\title{
Asymptotically Optimal Contextual Bandit Algorithm Using Hierarchical Structures
}

\author{
Mohammadreza Mohaghegh Neyshabouri ${ }^{\circledR}$, Kaan Gokcesu ${ }^{(}$, Hakan Gokcesu ${ }^{(}$, \\ Huseyin Ozkan, and Suleyman Serdar Kozat, Senior Member, IEEE
}

\begin{abstract}
We propose an online algorithm for sequential learning in the contextual multiarmed bandit setting. Our approach is to partition the context space and, then, optimally combine all of the possible mappings between the partition regions and the set of bandit arms in a data-driven manner. We show that in our approach, the best mapping is able to approximate the best arm selection policy to any desired degree under mild Lipschitz conditions. Therefore, we design our algorithm based on the optimal adaptive combination and asymptotically achieve the performance of the best mapping as well as the best arm selection policy. This optimality is also guaranteed to hold even in adversarial environments since we do not rely on any statistical assumptions regarding the contexts or the loss of the bandit arms. Moreover, we design an efficient implementation for our algorithm using various hierarchical partitioning structures, such as lexicographical or arbitrary position splitting and binary trees (BTs) (and several other partitioning examples). For instance, in the case of BT partitioning, the computational complexity is only log-linear in the number of regions in the finest partition. In conclusion, we provide significant performance improvements by introducing upper bounds (with respect to the best arm selection policy) that are mathematically proven to vanish in the average loss per round sense at a faster rate compared to the state of the art. Our experimental work extensively covers various scenarios ranging from bandit settings to multiclass classification with real and synthetic data. In these experiments, we show that our algorithm is highly superior to the state-of-theart techniques while maintaining the introduced mathematical guarantees and a computationally decent scalability.
\end{abstract}

Index Terms-Adversarial, big data, contextual bandits, multiclass classification, online learning, universal.

\section{INTRODUCTION}

$\mathbf{W}$ E STUDY online learning [1], [2] in the contextual multiarmed bandit setting [3]-[8]. In the classical formulation of the multiarmed bandit problem, one of the

Manuscript received November 8, 2017; revised June 4, 2018; accepted July 3, 2018. Date of publication August 2, 2018; date of current version February 19, 2019. This work was supported by the Turkish Academy of Sciences Outstanding Researcher Programme, Scientific and Technological Research Council of Turkey (Türkiye Bilimsel ve Teknolojik Arastirma Kurumu), under Contract 113E517. (Corresponding author: Mohammadreza Mohaghegh Neyshabouri.)

M. Mohaghegh Neyshabouri and S. S. Kozat are with the Department of Electrical and Electronics Engineering, Bilkent University, 06800 Ankara, Turkey (e-mail: mohammadreza@ee.bilkent.edu.tr; kozat@ee.bilkent.edu.tr).

$\mathrm{K}$. Gokcesu is with the Department of Electrical Engineering and Computer Science, Massachusetts Institute of Technology, Cambridge, MA 02139 USA (e-mail: gokcesu@mit.edu).

H. Gokcesu is with the School of Computer and Communication Sciences, École Polytechnique Fédérale de Lausanne, 1015 Lausanne, Switzerland (e-mail: hakan.gokcesu@epfl.ch).

H. Ozkan is with the Faculty of Engineering and Natural Sciences, Sabanc University, 34956 Istanbul, Turkey (e-mail: hozkan@sabanciuniv.edu).

Color versions of one or more of the figures in this paper are available online at http://ieeexplore.ieee.org.

Digital Object Identifier 10.1109/TNNLS.2018.2854796 available $M$ bandit arms (or actions) is chosen at each round to obtain a reward (or loss), and the reward (or loss) of all of the other unchosen $M-1$ arms stays oblivious. The objective is to maximize the cumulative reward of the selected arms in a series of rounds. Since the reward we would obtain from the other arms remains hidden, this setting can be considered as a limited feedback version of prediction with expert advice [9]-[14]. In addition, the well-known fundamental tradeoff between exploration and exploitation [15], [16] naturally appears in the multiarmed bandits. One should balance the exploitation of actions that gave the highest payoffs in the past and the exploration of actions that might give higher payoffs in the future.

The multiarmed bandit problem has attracted significant attention due to the applicability of the bandit setting in a wide range of applications from online advertisement [17] and recommender systems [18]-[20] to clinical trials [21] and cognitive radio [22], [23]. For example, in the online advertisement application, different advertisements available to display to users are modeled as the bandit arms, and the act of clicking by the user on the displayed advertisement is modeled as the reward [17].

In many instances of the bandit algorithms, additional information is available [24], such as the age or the gender of the patient in clinical trials [25], which is useful about the arm selection decision. However, most of the conventional bandit algorithms do not exploit or fail to fully exploit this information [26]-[28]. To remedy, contextual multiarmed bandit algorithms are introduced [16], [17], [29], where the additional information is represented as a context vector. For example, in the online advertisement applications, this context vector may contain certain information about the users, such as historical activities or demographic/geographical information. Then, the goal of the multiarmed bandit problem is extended to maximally exploit this additional information, i.e., the context, for optimizing the arm selection strategy and, therefore, gaining more rewards (or suffering less loss).

We consider the contextual extension in the online setting, where we operate sequentially on a stream of observations from a possibly nonstationary, chaotic, or even adversarial environment [30]-[32]. Hence, we have no statistical assumptions on the context vectors and behavior of the bandit arms so that our results are guaranteed to hold in an individual sequence manner [16]. We follow a competitive algorithm perspective [16] and define the performance (total time accumulated reward or loss) with respect to a competition class of context-dependent bandit arm selection policies. For this 
purpose, we design an exponentially large and parameterized competition class of predetermined mappings from the space of context vectors to the bandit arms, such that the best arm selection policy ${ }^{1}$ can be approximated arbitrarily well to a desired degree by the optimal mapping in the competition class. We point out that each mapping in our competition class partitions the space of context vectors into several disjoint regions and assigns each one of these regions to one of the bandit arms, i.e., each mapping selects the bandit arm corresponding to the region containing the observed context vector. Based on this competition class of such mappings, our goal is to asymptotically - at least-achieve ${ }^{2}$ the performance of the optimal mapping as well as the performance of the best arm selection policy at a faster convergence (performancewise or in terms of the convergence of the regret upper bound to zero) rate compared to the state of the art as more data are observed.

In order to generate partitions of the context space and, therefore, a rich competition class, we use various hierarchical partitioning structures [33], such as the ones based on lexicographical or arbitrary position splitting, binary trees (BTs), and several other partitioning examples (see Section IV). In our design, each of these structures leads to a different competition class but approximates (arbitrarily well, and even perfectly if desired) the same best arm selection policy by the optimal mapping in the corresponding competition class. However, each hierarchical structure encodes the best arm selection policy differently and one of them is the most efficient in the sense of the required number of partition regions (i.e., less number of regions means higher efficiency). Therefore, we explore various hierarchical structures and introduce an algorithm that covers each of such structures by using a carefully designed weighting over the corresponding competition class. The output of the introduced algorithm is the optimal data adaptive combination (with respect to the designed weighting) of the policies (aforementioned mappings) in the competition class. Our weighting/adaptive combination favors simpler models in the beginning of the data stream and gradually switches to more complex ones as the data overwhelm.

As a result, our algorithm is guaranteed to asymptotically perform - at least—as well as the best arm selection policy. We achieve this performance optimality at a faster convergence rate [for instance, at the rate $O\left(((R M \ln M \ln N) / T)^{1 / 2}\right)$ in the case of BT partitioning after averaging the regret bound over $T$, where $R$ is the number of regions in the optimal partition, $M$ is the number of bandit arms, $N$ is the number of regions in the finest partition in the competition class, and $T$ is the number of rounds] compared to the state-of-the-art ${ }^{3}$

\footnotetext{
${ }^{1}$ This best arm selection policy is based on the fixed best partitioning of the context space and the best assignment of the arms to the regions of that best partition. It is not necessarily in our competition class. However, it can be approximated arbitrarily well by the optimal mapping in the class by varying the class parameter, and it can be determined only when the complete data stream is observed.

${ }^{2}$ In addition to achieving, we might well outperform since our approach is data driven and based on combination of partitions, i.e., we do not rely on a single fixed partition.

${ }^{3}$ The convergence rates given here samples our general regret results (after averaging over $T$ ) in the case of BT partitioning. Our rates for other partitionings in our generic class of hierarchical structures naturally vary, but our superiority compared to the state of the art stays valid in a similar manner (see Section IV, for our complete regret results for all structures).
}

rate $O\left(((M N \ln M) / T)^{1 / 2}\right)$. Note that here, typically, $N \gg R$ is the dominating factor. Our superior performance is due to exploiting the right hierarchical partitioning structure that encodes the best policy more efficiently and, therefore, assigns higher initial weights to the optimal partition. This exploitation of the right structure with the introduced weighting scheme also mitigates the overfitting issue as an additional merit.

We emphasize that our algorithm is designed to work for a generic class of hierarchical partitioning structures and our optimality results do hold for each type of structure in this generic class. Therefore, one can use the proposed algorithm with any type of partitioning that is appropriate for the target application with the corresponding performance guarantees. Such guarantees include upper bounds on the regret with respect to the best arm selection policy that is mathematically proven to vanish at $O(1 / \sqrt{T}$ ) (after averaging over $T$ ) in a superior manner over the state of the art (see Sections I-A and IV, for detailed comparisons). We also present a computationally high-efficient implementation for the introduced algorithm that, for instance, combines $M^{N}$ mappings with only computational complexity of $O(M \ln N)$ in the case of BT partitioning structure. Through an extensive set of experiments with real and synthetic data, we demonstrate the proposed approach in several scenarios, such as multiclass classification, online advertisement, and multiarmed bandit along with various partitioning structures. In these experiments, our algorithm is shown to significantly outperform the state-of-the-art techniques with real-time data processing and strong modeling capabilities.

\section{A. Prior Art}

The contextual bandit problem is mostly studied in the stochastic setting [29], [34], [35], where context vectors and losses are assumed to be drawn randomly and independently of an unknown distribution. Additional assumptions regarding the relations between the context vectors and the arm losses are also used in other studies, e.g., a linear relation in [17] and [36], and more general ones in [37]. These algorithms essentially fail to hold their performance guarantees if the context vectors or the arm losses are chosen by an adversary rather than by a prefixed distribution.

An alternative to the stochastic approaches is the adversarial setting, where algorithms do not use any assumptions on the behavior of the context vectors and bandit arms. The wellknown EXP3 algorithm [32] formulates the noncontextual bandit problem in an adversarial setting and achieves a regret upper bound ${ }^{4}$ of $O\left((T M \ln M)^{1 / 2}\right)$ against the best arm. $S$-EXP3 algorithm [16] is a naive extension of EXP3 in the contextual setting, which partitions the context space and runs independent EXP3 algorithms over each one of the partition regions. S-EXP3 achieves a regret upper bound of $O\left((T N M \ln M)^{1 / 2}\right)$ against the best mapping from the regions to the bandit arms, where $N$ is the number of regions in the partition of the context space. As implied by the regret bound, the $S$-EXP3 algorithm works well only when the

\footnotetext{
${ }^{4}$ We illustrate regret upper bounds without averaging over $T$ here in this section, but with averaging in Section I to demonstrate the convergence to 0 there.
} 
complexity (the granularity or the level of detailing/fineness) of the required partitioning to model the truly optimal selection policy is relatively small, otherwise it quickly overfits and suffers from insufficient data.

The EXP4 algorithm [32] is another extension of EXP3 in the contextual setting. In this algorithm, a set of $K$ experts observe the context vectors and suggest distributions on the arms. Their suggestions are adaptively combined to select the arm to pull. It is shown that EXP4 achieves a regret upper bound of $O\left((T M \ln K)^{1 / 2}\right)$ against the best expert. Considering the $M^{N}$ mappings from a partition of the context space to the arms as the $K$ experts, EXP4 achieves $O\left((T N M \ln M)^{1 / 2}\right)$ against the optimal mapping. As we show in Section III, the EXP4 algorithm can be improved by producing an initial tendency (in earlier times of the stream) toward the mappings of smaller complexity. In this case, although the finest partition has $N$ regions (and hence, there are $M^{N}$ mappings in total), it suffices to run EXP4 over $O\left((N M)^{R}\right)$ mappings with $R$ regions, resulting a regret bound of $O\left((T M R \ln (N M))^{1 / 2}\right)$, if the optimal partition consists of $R$ regions. However, the main problem with this algorithm is its computational complexity of $O\left((N M)^{R}\right)$. On the other hand, the contextual semi bandit-Follow the Perturbed Leader (CSB-FTPL) algorithm [38] achieves a regret upper bound of $O\left(T^{2 / 3} M(\ln K)^{1 / 2}\right)$ against the best expert among a set of $K$ experts with a computational complexity that is polynomial in $\ln K$. Hence, running $C S B$-FTPL over $O\left((N M)^{R}\right)$ mappings with $R$ disjoint regions yields a regret upper bound of $O\left(T^{2 / 3} M(R \ln N)^{1 / 2}\right)$ with a polynomial computational complexity in $\ln N$.

We emphasize that we seek to achieve a regret upper bound vanishing (with respect to the rounds/time after averaging over $T$ ) faster than that of EXP4 with a computational complexity linear in $\ln N$, which allows us to grow the hierarchical structure freely. To this end, our algorithm not only drastically reduces the computational complexity (e.g., down to $O(M \ln N)$ in the case of BT partitioning) compared to the discussed state-of-the-art techniques but also achieves a regret upper bound of $O\left((T M R \ln M \ln N)^{1 / 2}\right)$.

Finally, a simple instance of our hierarchical structures, the context trees, is widely used in various applications, including, but not limited to, data compression [39], [40], estimation [41], [42], communications [43], regression [44], [45], and classification [46]. In all aforementioned applications, context trees are used to partition the context space in a nested structure, run an independent adaptive model over each one of the tree nodes, and combine the models. On the other hand, in this paper, we use a generalized novel notion of hierarchical structures that are specifically designed for the completely different multiarmed contextual bandit problem.

\section{B. Contributions}

Our main contributions are as follows:

1) We introduce a novel and efficient contextual bandit arm selection algorithm that first quantizes the space of context vectors and, then, achieves the performance of the optimal mapping from the quantized regions to the bandit arms (in the average loss per round sense).

2) We introduce an efficient quantization method and show that using this quantization method, our algorithm asymptotically achieves (not only the optimal mapping but also) the performance of the best arm selection policy (in the average loss per round sense) as the number of quantization levels increases.

3) We introduce a novel and generalized notion of hierarchical context space partitioning structures for the contextual bandit setting and use such hierarchical structures to design an efficient implementation of our algorithm and achieve a faster convergence rate for the regret compared to the state of the art.

4) We demonstrate significant performance gains with the proposed algorithm in comparison to the state-of-the-art techniques through extensive experiments involving both synthetic and real data.

\section{Organization of this Paper}

In Section II, we describe the contextual multiarmed bandit framework. Next, we explain a first mixture of expert-based approach and its challenges in Section III. In Section IV, we explain the notion of hierarchical structures and implement our algorithm using these structures. We introduce an efficient quantization method in Section $\mathrm{V}$ and show that our algorithm is competitive against any mapping, including the best arm selection policy, from the context space to the bandit arms. Section VI contains the experimental results over several synthetic and well-known real-life data sets followed by the concluding remarks in Section VII.

\section{Problem Description}

We study the contextual bandit problem in an adversarial setting. ${ }^{5}$ Recall that the original multiarm bandit problem is a sequential game. One of the available bandit arms $I_{t} \in\{1, \ldots, M\}$ is selected at each round $t$, and then, a related loss $l_{t, I_{t}}$ is observed. ${ }^{6}$ The objective is to minimize the accumulated loss $\sum_{t=1}^{T} l_{t, I_{t}}$ in a sequence of $T$ rounds. In the contextual extension, a context vector $\mathbf{s}_{t}$ from a context space $S$ is additionally provided at each round before selecting the arm. For example, $S$ is $[0,1]^{2}$ in Fig. 1. Then, the objective stays same but can be improved with the available context.

We consider this contextual bandit problem in adversarial setting [47], where at each round $t$, an adversary assigns a specific loss to each arm $i \in\{1,2, \ldots, M\}$ simultaneously in parallel with the player who chooses an arm to pull. The adversary's goal is to maximize the player's loss, whereas the player tries to maximize her/his gain (here, the loss maximization by the opponent gives the name "adversary"). We emphasize that the adversary is provided with all the information from the previous rounds. It can even know the algorithm followed by the player. However, if the player's choice is randomized, then the adversary does not know the outcome of this randomization while assigning the losses to

\footnotetext{
${ }^{5}$ All vectors are column vectors and denoted by boldface lower case letters. For a $K$-element vector $\mathbf{u}, u_{i}$ represents the $i$ th element and $\|\mathbf{u}\|=\left(\mathbf{u}^{T} \mathbf{u}\right)^{1 / 2}$ is the $l^{2}$-norm, where $\mathbf{u}^{T}$ is the transpose. Indicator function $\mathbf{1}_{\{\cdot\}} \in\{0,1\}$ outputs 1 only if its argument condition holds. A function $f: \mathbb{R}^{n} \rightarrow \mathbb{R}$ is Lipschitz continuous over a region $W \subset \mathbb{R}^{n}$ if there exists a nonnegative constant $c$, such that $\left|f\left(\mathbf{x}_{1}\right)-f\left(\mathbf{x}_{2}\right)\right| \leq c\left\|\mathbf{x}_{1}-\mathbf{x}_{2}\right\|$ for all $\mathbf{x}_{1}, \mathbf{x}_{2} \in W$.

${ }^{6}$ We assume $l_{t, I_{t}} \in[0,1]$ for simplicity; however, it can be straightforwardly shown that our results hold for any bounded loss after shifting and scaling in magnitude.
} 


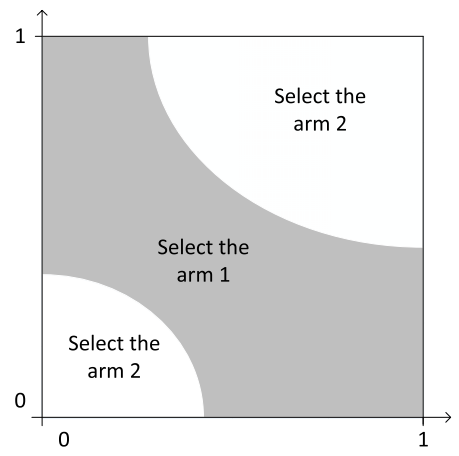

(a)

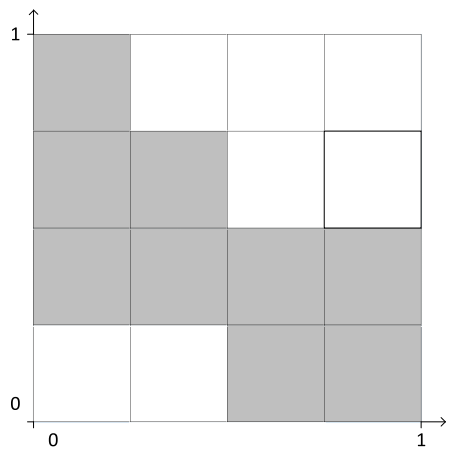

(b)

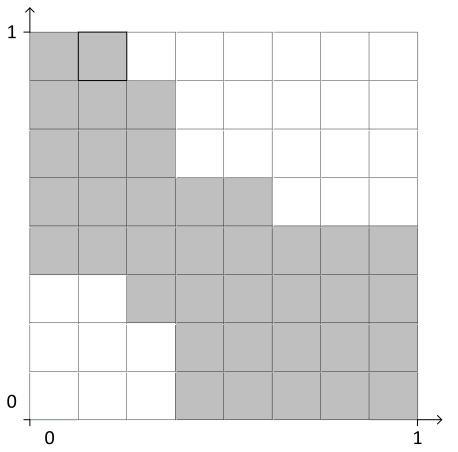

(c)

Fig. 1. Example mapping from the context space to the set of bandit arms and its approximations in the quantized competition classes. In each mapping, the dark and bright sections are mapped to the arms 1 and 2, respectively. (a) Example mapping from the context space [0, 1$]^{2}$ to the set of bandit arms $\{1,2\}$. (b) Closest mapping in the quantized competition class with 16 quantization levels to the mapping in (a). (c) Closest mapping in the quantized competition class with 64 quantization levels to the mapping in (a).

the arms, e.g., the adversary may know that the player tosses a coin to choose the arm to pull but does not know the outcome of the toss. Namely, "adversarial setting" refers to the algorithmic framework or the game, in which the data generation (assignment of losses in this case) or the adversary is acting against the player on purpose, while the player tries to maximize her/his gain. In accordance with the nature of this adversarial setting, in designing the algorithm for the player to use, we make no statistical assumptions about the context vectors and the bandit arms [32], and our performance bounds are guaranteed to hold in an individual sequence manner. Hence, in designing our algorithm, we rigorously address such adversarial conditions and provide strong mathematical guarantees that hold for all possible data streams or for all possible moves of the adversary. Our algorithm is strictly sequential, such that at each round $t$, it selects an arm $I_{t}$ according to the information coming from the previous rounds, including observed context vectors, selected arms, and their losses; alongside the context vector that we are currently observing, i.e.,

$$
I_{t}=f_{t}\left(\mathbf{s}_{t} ; \mathbf{s}_{t-1}, I_{t-1}, l_{t-1, I_{t-1}} ; \ldots ; \mathbf{s}_{1}, I_{1}, l_{1, I_{1}}\right) .
$$

In design of our algorithm, we aim at sequentially learning the optimal partitioning of the context space with the optimal assignment between the regions of the learned partition and the set of arms. For this purpose, we investigate a general framework of hierarchical structures to generate context space partitions and eventually learn the asymptotically optimal, time varying, context-driven arm chooser $f_{t}$. We show that our approach, compared to the state-of-the-art techniques, yields a computationally highly superior algorithm with real-time data processing capabilities while achieving a faster convergence rate to the optimal conditions (in terms of the convergence of the regret upper bounds to 0 ). The superiority of the proposed algorithm is due to that the set of all possible context space partitions considered here can theoretically achieve arbitrarily high degree of granularity (can be of arbitrarily high capacity), whereas the true complexity of the optimal partition is limited (see Section IV) in reality. Based on this observation, our approach additionally allows the regret analysis to incorporate an upper bound on the complexity of the optimal partition, which in turn significantly improves the convergence of the presented algorithm in almost all practical scenarios. This gain is essentially from $O(\sqrt{N})$ to $O\left((\ln N)^{1 / 2}\right)$ [N is measuring the granularity (see Section IV)]. If the complexity of the optimal partition cannot be upper bounded, which would be a purely theoretical consideration as the true complexity is almost always limited and finite in real scenarios, our regret analysis then produces similar rates of convergence in that very worst theoretical scenario. Nevertheless, in any case, the proposed algorithm is computationally highly efficient and superior and asymptotically optimal in the adversarial setting, including the very worst scenario regardless of the stationary or nonstationary or perhaps chaotic source statistics.

To this end, we consider a large class $\mathcal{G}$ of deterministic mappings, i.e., $\forall g \in \mathcal{G}, g: S \rightarrow\{1, \ldots, M\}$. Each such mapping is composed of a fixed partition of the context space, and an arm is assigned to each partition region. Depending on the partition region that a context $s_{t}$ falls in, $g$ chooses the assigned arm $g\left(s_{t}\right)$. An example is shown in Fig. 1(a) in the case of $2-\mathrm{D}$ context space $S=[0,1]^{2}$ with 2 bandit arms, where $g\left([0.5,0.5]^{T}\right)=1$. Note that for a given $g \in \mathcal{G}$, all of the other deterministic mappings resulting from all possible arm assignments to the regions of the partition of $g$ are also included in $\mathcal{G}$. Since we work in the adversarial setting and therefore refrain from making any statistical assumptions about the context vectors and the loss of the bandit arms [32], we next define our performance with respect to the optimum (minimum loss) mapping in the "competition" class $\mathcal{G}$ based on the following regret:

$$
\mathcal{R}(T, \mathcal{G}) \triangleq \max _{g \in \mathcal{G}} \mathbb{E}\left[\sum_{t=1}^{T} l_{t, I_{t}}-\sum_{t=1}^{T} l_{t, g\left(\mathbf{S}_{t}\right)}\right]
$$

where the expectation is with respect to the internal randomization in our algorithm (the internal randomization here is not related to data statistics). Our goal is to upper bound the regret by a term that depends sublinearly in $T$ and, hence, asymptotically achieve-at least - the performance of the best $g$ in $\mathcal{G}$ (in the averaged regret per round sense). 
Achieving this goal is equivalent to achieving the performance of the chooser of the optimal context space partition with the optimal assignment to the arms. Here, optimality of the context space partition should be understood with respect to the class $\mathcal{G}$, which is certainly not restrictive, since it can be arbitrarily improved by generalizing (detailing) $\mathcal{G}$ to a desired degree (see Section III).

We next construct the class $\mathcal{G}$ and provide a mixture-ofexpert-based first solution to the introduced problem.

\section{Contextual Bandit Algorithm BASED ON MIXTURE OF EXPERTS}

The ultimate goal in the contextual bandit problem is ideally to achieve the performance of the best mapping in the set $\mathcal{U}^{7}$ of all arbitrary mappings from the context space to the bandit arms. Since this set of all arbitrary mappings is too powerful to compete against in design of an algorithm, as the first step, we uniformly quantize the context space $S$ into $N$ disjoint regions $r_{1}, r_{2}, \ldots, r_{N}$, i.e., $\cup_{i=1}^{N} r_{i}=S$ and $r_{i} \cap r_{j}=\varnothing$ for $\forall i \neq j$. We use uniform quantization for simplicity, however, one can incorporate any arbitrary type of quantization into our framework straightforwardly. In our framework, we consider all possible assignments between the set of disjoint regions and the set of bandit arms and call each context mapping resulting from one of those assignments an $N$-level quantized mapping. Therefore, each $N$-level quantized mapping is essentially a function from $\cup_{i=1}^{N} r_{i}=S$ to $\{1, \ldots, M\}:$ a context $s \in r^{*} \subset S$ is mapped to the bandit arm that the region $r^{*}$ is assigned to. Two examples of such quantized mappings of different levels for the case of 2-armed bandit with the context space $[0,1]^{2}$ are shown in Fig. 1(b) and (c). Given a quantized context space $S=\cup_{i=1}^{N} r_{i}$, we define the class $\mathcal{G}^{N}$ of $N$-level quantized mappings as the "competition class" with $N$ quantization levels consisting of all arbitrary assignments between the bandit arms and the given $N$ regions $\left\{r_{i}\right\}_{i=1}^{N}$.

Remark: We seek to achieve the performance of the best quantized mapping in $\mathcal{G}^{N}$, which can get arbitrarily close (and $N$ can be freely chosen in our framework) to the performance of the best arbitrary mapping in $\mathcal{U}$, i.e., the best arm selection policy, as $N$ increases. For example, suppose that the mapping shown in Fig. 1(a) is the best arbitrary mapping. In this case, the mappings in Fig. 1(b) and (c) of improving optimalities will be the best mappings in $\mathcal{G}^{16}$ and $\mathcal{G}^{64}$, respectively.

Based on $M^{N}$ different mappings in $\mathcal{G}^{N}$, we consider an expert chooser that is one-to-one corresponding to each of those mappings, such that $g_{j}(s)$ is the arm chosen by expert $E_{j}$ for the context $s$, i.e., $E_{j} \leftrightarrow g_{j}, 1 \leq j \leq M^{N}$. An example of all 16 mappings followed by the experts for the case of $M=2$ and $N=4$ is shown in Fig. 2, where, unlike Fig. 1, we choose a nonuniform quantization to demonstrate the generality in our approach. One of these experts in Fig. 2 is $\mathcal{G}^{4}$-optimal for the underlying sequence of losses, however, naturally, we do not know which. Hence, instead of committing to a single expert, we next use a mixture of experts approach to learn the best one during rounds.

\footnotetext{
${ }^{7}$ This set $\mathcal{U}$ consists of all possible arbitrary context space partitions (not confined to $\mathcal{G}$ ) with all possible assignments of partition regions to the arms.
}

1)

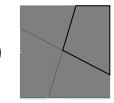

2)

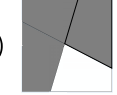

3)

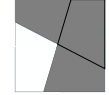

4)

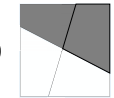

5)

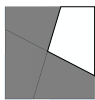

6)

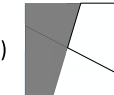

7)

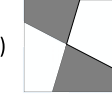

8)

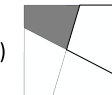

9)

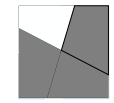

10)

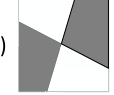

11)

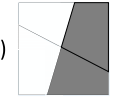

12)

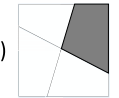

13)

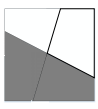

14)

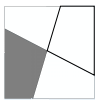

15

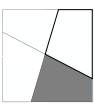

16)

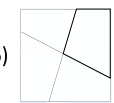

Fig. 2. All possible mappings in a 2-armed bandit problem with a predetermined quantization of the context space $S=[0,1]^{2}$ into four regions. In each mapping, the dark and bright regions are mapped to the arms 1 and 2, respectively.

In order to achieve the performance of the best expert, we assign each expert $E_{j}$ a weight $\alpha_{t, j}$ (showing our trust on the expert $E_{j}$ at round $t$ ) and use exponentiated weights to adaptively combine them. After observing context $\mathbf{s}_{t}$ at each round $t$, we randomly select one of the experts using the probability simplex $\boldsymbol{\beta}_{t}=\left(\beta_{t, 1}, \ldots, \beta_{t, M^{N}}\right)$, where $\beta_{t, j}=\alpha_{t, j} / \sum_{k=1}^{M^{n}} \alpha_{t, k}$ is the normalized weight. Importantly, the probability of selecting each arm then follows the probability simplex $\mathbf{p}_{t}=\left(p_{t, 1}, \ldots, p_{t, M}\right)$, where

$$
p_{t, i}=\sum_{j=1}^{M^{N}} \beta_{t, j} \mathbf{1}_{\left\{g_{j}\left(\mathbf{s}_{t}\right)=i\right\}} .
$$

We initially set the weights $\alpha_{1, i}$ according to the complexity of the mappings of experts from $\mathcal{G}^{N}$ and use exponentiated losses to update during rounds; at each round $t \geq 2$, we have

$$
\alpha_{t, i}=\alpha_{1, i} e^{-\eta \sum_{\tau=1}^{t-1} \tilde{l}_{\tau, g_{i}}\left(\mathbf{S}_{\tau}\right)}
$$

where $\eta \in \mathbb{R}^{+}$is the (constant) learning rate and $\tilde{l}_{\tau, g_{i}\left(\mathbf{s}_{\tau}\right)}$ is

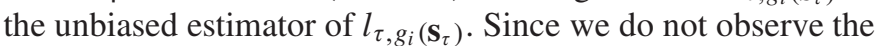
loss $l_{t, m}$ of the unchosen arms, we use the unbiased estimator

$$
\tilde{l}_{t, m}= \begin{cases}\frac{l_{t, m}}{p_{t, m}}, & m=I_{t} \\ 0, & m \neq I_{t}\end{cases}
$$

where $\mathbb{E}\left[\tilde{l}_{t, m}\right]=l_{t, m}$. Using this bandit arm selection probability assignment defined through (3)-(5), we have the following regret result.

Theorem 1: Consider an $M$-armed contextual bandit problem. If the context space is quantized into $N$ disjoint regions, and experts $E_{j}$ 's are following the $M^{N}$ possible mappings in $\mathcal{G}^{N}$, as described in Section III, then $\mathcal{R}\left(T, E_{j}\right)$ satisfies

$$
\mathcal{R}\left(T, E_{j}\right) \leq \frac{\ln \left(1 / \beta_{1, j}\right)}{\eta}+\frac{M T \eta}{2}
$$

based on the probability assignments defined through (3)-(5), where $T$ is the number of rounds, $\eta \in \mathbb{R}^{+}$is the learning rate parameter in (4), and $\beta_{1, j}$ is the normalized initial weight of the $j$ th expert $E_{j}$. 
Proof of Theorem 1 follows similar lines to [16, Proof of Th. 4.2] with certain variations due to our arbitrary initial weighting as opposed to uniform initial weights of the experts in [16]. The proof of our Theorem 1 is provided in Appendix A.

We observe that the regret bound is logarithmically dependent on the reciprocal of the prior weight of the optimal partitioning in the competition class (i.e., its complexity cost). Hence, by using equal prior weights on the $M^{N}$ experts, our regret bound will be in the $\operatorname{order}^{8}$ of $O(\sqrt{N T})$ (after optimizing the learning rate). We point out that this result is similar to the EXP4 algorithm [16] that achieves a regret upper bound of $O(\sqrt{N T})$ with optimum selection of the learning rate. Furthermore, S-EXP3 algorithm [16] achieves a regret upper bound of the same order $O(\sqrt{N T})$ using an independent EXP3 algorithm over each quantized region of the context space. This square-root dependence of the regret bound on the quantization level is prohibitive and working against our motivation of approximating the performance of the best arbitrary mapping by freely increasing the number of quantization levels. Instead, we would like our regret bound to be dependent on the actual number $R$ of disjoint regions that are needed and sufficient to model the actual complexity of the best arbitrary mapping, whatever the quantization level $N$ is. Hence, we want to achieve the order $O(\sqrt{R T})$. Moreover, working with these $M^{N}$ parameters $\alpha_{t, 1}, \ldots, \alpha_{t, M^{N}}$ has quite high space and computational complexities of $O\left(M^{N}\right)$.

To this end, we introduce hierarchical structures to generate context space partitions and exploit the level of complexity that is sufficient to model the best mapping over the introduced hierarchy. Thus, we achieve a regret upper bound with squareroot dependence on the actual number of regions $R$ in a computationally highly superior manner with significantly low space complexity.

\section{Hierarchical Structures}

We use hierarchical structures to implement our contextual bandit algorithm efficiently in terms of both the regret upper bound convergence to 0 in average loss per round sense as well as computational and space complexities. Suppose that we have $H$ nodes in a hierarchical structure labeled $v_{i}$, $i \in\{1,2, \ldots, H\}$. We assign each node $v_{i}$ a region $r_{i}$ from the context space, and there is a hierarchical connection from each parent node to its child nodes. Let $\Phi_{i}$ be the set of child node groups of the node $v_{i}$, where each group $\phi \in \Phi_{i}$ consists of child nodes, such that the union of their corresponding regions gives the region associated with the parent node $v_{i}$.

For instance, consider the BT of depth 2 in Fig. 3, which quantizes the 2-D context space $S=[0,1]^{2}$. Each node of such BT corresponds to a region of the context space, as shown in Fig. 3. The region corresponding to each node is the union of the regions of its child nodes. Hence, for each node $v_{i}$ in this tree (except for the leaf nodes), the set $\Phi_{i}$ is of size 1, which consists of only one group of cardinality 2 (which is the

\footnotetext{
${ }^{8}$ For ease of exposition and simplicity in our order notation here, we drop the variables, on which the dependence of order is similar or same or negligible across the compared algorithms.
}

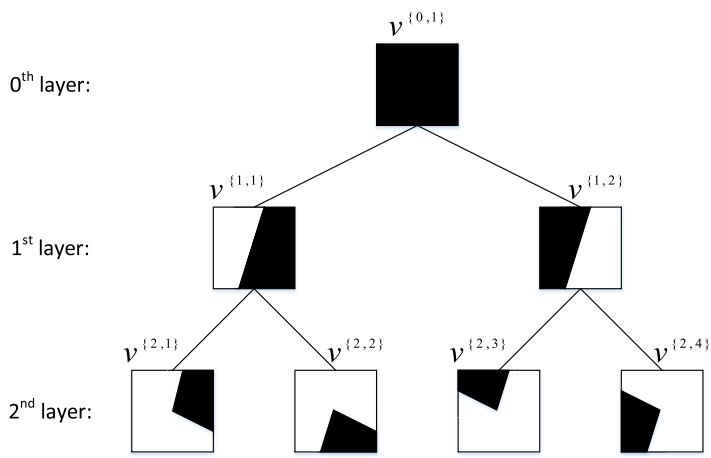

Fig. 3. BT of depth $D=2$ over the context space $[0,1]^{2}$. The regions corresponding to each node are filled with black.

parent node's child pair). For the leaf nodes, $\Phi_{i}$ is the empty set and, hence, has a size of 0 .

Next, we use this hierarchical structure to compactly represent our experts and combine them in an efficient manner.

\section{A. Weighted Mixture of Experts Algorithm Using Hierarchical Structures}

In the following, we explain the details of our efficient implementation of the mixture of experts algorithm (described in Section III) by using the hierarchical structures and present several examples. In addition to achieving computational scalability in our implementation, another goal of this paper is to incorporate the model complexity of the best expert to improve the upper bound on the regret.

Here, each expert is composed of a partition of the context space and an arm assigned to each partition region. The partition corresponding to each expert can be represented using several nodes of the hierarchical structure. Hence, each expert can be represented using several nodes (showing the partition) and an arm corresponding to each one of them (showing the arm assignments). As an example, consider a 2 -armed bandit problem. Suppose that we use a BT of depth 2 to quantize the context space into four regions. In this case, we define $2^{4}=16$ experts as in Fig. 2. We represent four samples among these 16 experts on our BT in Fig. 4. In Fig. 4, the nodes representing the partition corresponding to the experts are marked using the circles and the arm selected by the expert at each one of these nodes is declared over the node. We seek to adaptively combine all of the experts to achieve the performance of the best one, as explained in Section III.

In order to implement our mixture of experts, over each node $v_{i}$, we define $M$ parameters $\alpha_{t, m, i}$ for $m=1$ to $M$ as the weight of $m$ th arm in the node $v_{i}$. This weight shows our trust on the $m$ th arm when the context vector falls into the region corresponding to the node $v_{i}$. We set $\alpha_{1, m, i}=1$ for all $m$ 's and $v_{i}$ 's, and for $t \geq 2$,

$$
\alpha_{t, m, i}=\exp \left(-\eta \sum_{\tau=1}^{t-1} \frac{l_{I_{\tau}}}{p_{\tau, m}} \mathbf{1}_{\left\{I_{\tau}=m\right\}} \mathbf{1}_{\left\{\mathbf{s}_{\tau} \in r_{i}\right\}}\right) .
$$

We can easily update these weights as follows. At each round $t$, after we receive $\mathbf{s}_{t}$, calculate $\mathbf{p}_{t}$, select $I_{t}^{\text {th }}$ arm, and observe the loss $l_{t, I_{t}}$, we calculate

$$
\alpha_{t+1, m, i}=\alpha_{t, m, i} \exp \left(-\eta \frac{l_{I_{t}}}{p_{t, m}} \mathbf{1}_{\left\{I_{t}=m\right\}} \mathbf{1}_{\left\{\mathbf{s}_{t} \in r_{i}\right\}}\right) .
$$




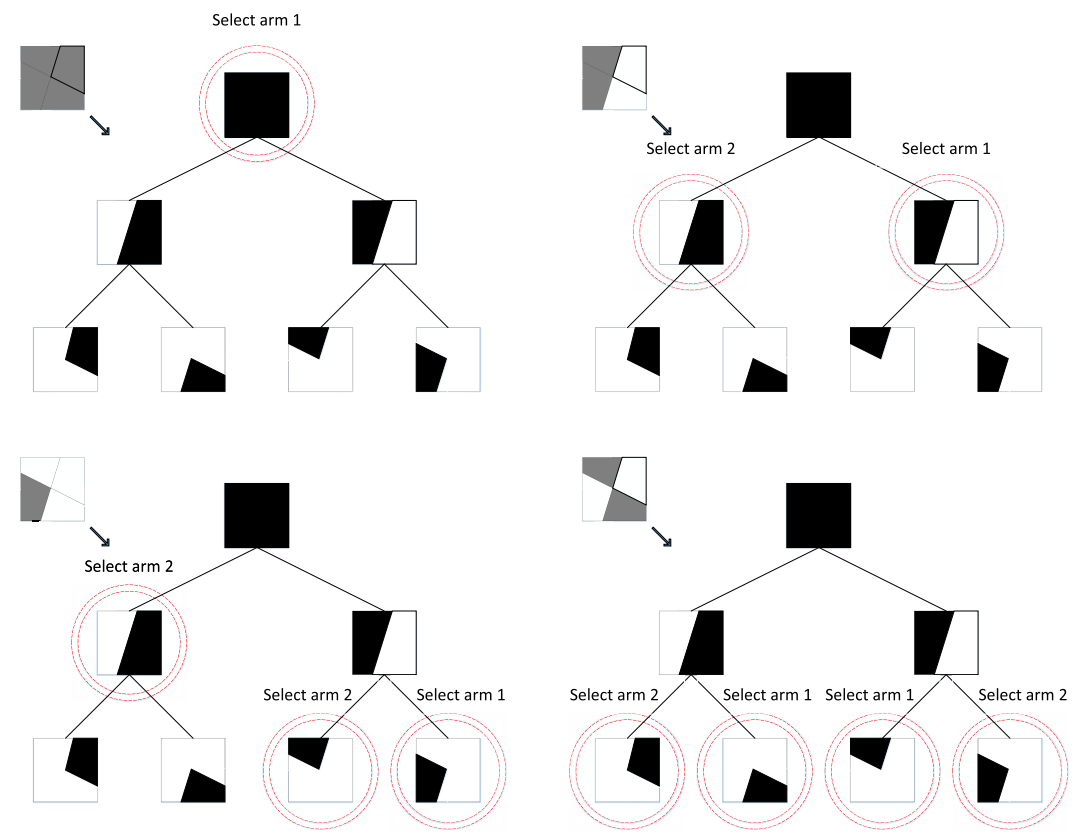

Fig. 4. Representation of four sample mappings in Fig. 2 over the BT in Fig. 3.

We point out that the weight of each expert $\alpha_{t, k}$ in (4) can be written as a multiplication of its initial weight and our weight parameters (i.e., $\alpha_{t, m, i}$ 's) on the tree nodes corresponding to the mapping followed by the expert. To this end, in order to obtain the expert weights (see Theorem 2), we define another variable $w_{t, i}$ over each node $v_{i}$, such that

$$
w_{t, i}=\frac{1}{\left(\left|\Phi_{i}\right|+1\right) M} \sum_{m=1}^{M} \alpha_{t, m, i}+\frac{1}{\left|\Phi_{i}\right|+1} \sum_{\phi \in \Phi_{i}}\left(\prod_{j \in \phi} w_{t, j}\right) .
$$

Hence, if $\Phi_{i}$ is the empty set (i.e., $\left|\Phi_{i}\right|=0$ ), then the equation simply becomes

$$
w_{t, i}=\frac{1}{M} \sum_{m=1}^{M} \alpha_{t, m, i} .
$$

The following proposition shows that using this recursion to calculate $w_{t, i}$ variables, the weight of the root node $w_{t, 1}$ becomes equal to the sum of the expert weights, i.e., $\sum_{k} \alpha_{t, k}$ [as defined in (4)].

Proposition 1: Using the recursive formula in (9), at each node $v_{i}$, we have

$$
w_{t, i}=\sum_{k \in \Gamma_{i}} \alpha_{t, k}
$$

where $\Gamma_{i}$ is the set of all experts defined over node $v_{i}$.

Proof of Proposition 1 is provided in Appendix B.

Now, in order to calculate the probability simplex in (3), we define $M$ other variables to calculate $\sum_{k} \alpha_{t, k} \mathbf{1}_{\left\{g_{k}\left(\mathbf{S}_{t}\right)=i\right\}}$ for $i=1, \ldots, M$. To this end, after we observe $\mathbf{s}_{t}$, we set

$$
\gamma_{t, m, i}=\frac{1}{M} \alpha_{t, m, i}
$$

at the nodes $v_{i}$ containing $\mathbf{S}_{t}$, where $\left|\Phi_{i}\right|=0$ (i.e., leaf nodes). Then, we go up on the hierarchy using a recursive formula similar to the way we calculate $w_{t, i}$ variables in (9) as

$$
\begin{aligned}
\gamma_{t, m, i}= & \frac{1}{\left(\left|\Phi_{i}\right|+1\right) M} \alpha_{t, m, i} \\
& +\frac{1}{\left|\Phi_{i}\right|+1} \sum_{\phi \in \Phi_{i}}\left(\prod_{j \in \phi} w_{t, j}\left(\frac{\gamma_{t, m, j}}{w_{t, j}}\right)^{\mathbf{1}_{\left\{\mathbf{S}_{t} \in r_{j}\right\}}}\right) .
\end{aligned}
$$

Using this recursion, we calculate $\gamma_{t, m, 1}$ for $m=1, \ldots, M$. The following proposition shows that using this recursion, $\gamma_{t, m, 1}$ is the weighted sum of all experts, which select the $m$ th arm when they observe $\mathbf{s}_{t}$. Hence, we can build the probability simplex in (3) as

$$
p_{t, m}=\gamma_{t, m, 1} / w_{t, 1} \quad \forall m \in\{1, \ldots, M\} .
$$

Proposition 2: Using the recursive formula in (13), at each node $v_{i}$ for all $m \in\{1, \ldots, M\}$, we have

$$
\gamma_{t, m, i}=\sum_{k \in \Gamma_{i}} \alpha_{t, k} \mathbf{1}_{\left\{g_{k}\left(\mathbf{s}_{t}\right)=m\right\}}
$$

where $\Gamma_{i}$ is the set of all experts defined over node $v_{i}$.

Proof of Proposition 2 is provided in Appendix C.

With the proposed implementation of the algorithm, at each round $t$, after observing $\mathbf{s}_{t}$, we first calculate $\gamma_{t, m, 1}$ for $m=1, \ldots, M$ and, then, divide by $w_{t, 1}$ to form the probability simplex $\mathbf{p}_{t}=\left(p_{t, 1}, \ldots, p_{t, m}\right)$, using which we select an arm $I_{t}$. After we select our arm and suffer the loss according to the selected arm, we first update $\alpha_{t, I_{t}, i}$ parameters at the nodes containing $\mathbf{s}_{t}$. Then, we update $w_{t, i}$ variables at these affected nodes and go to the next round. The pseudocode of the explained procedure is provided in Algorithm 1. 


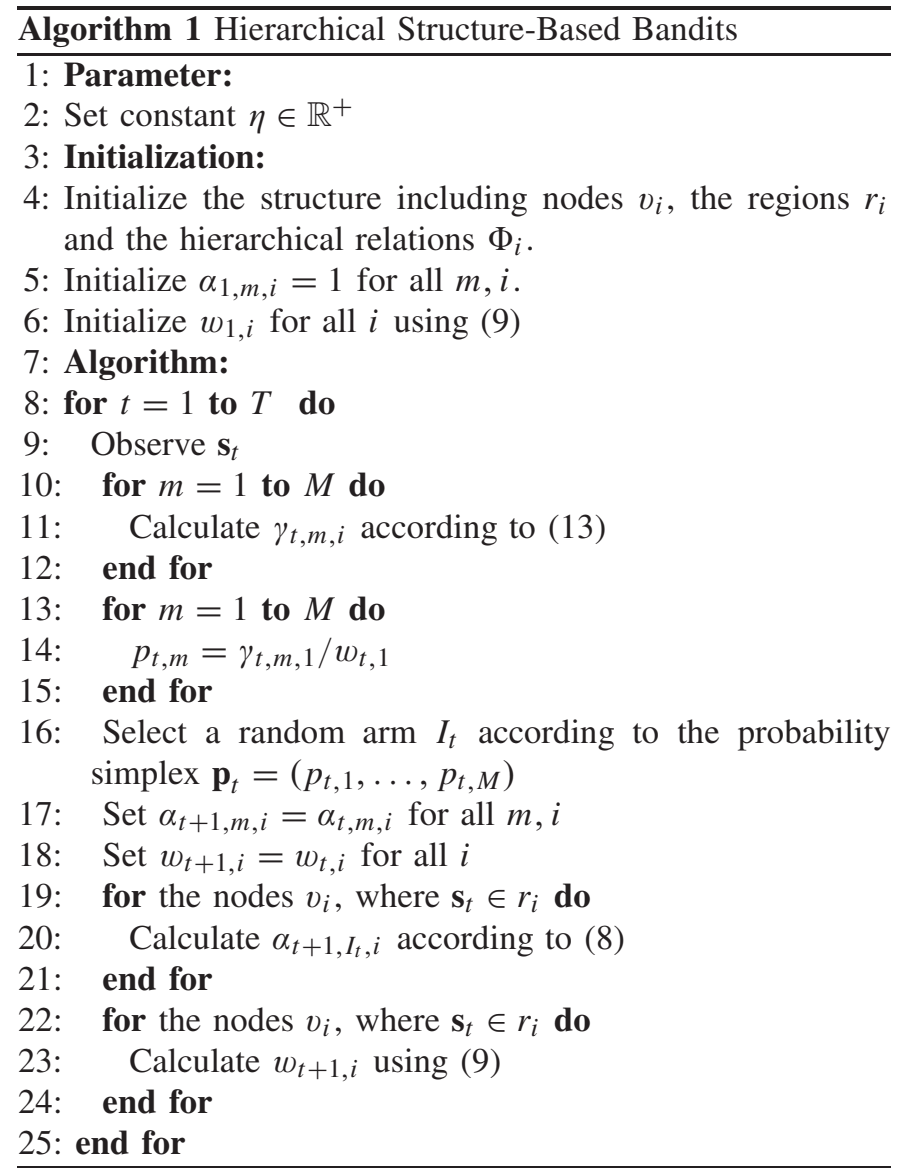

Next, we show the regret bound of our hierarchical structure algorithm.

Theorem 2: Algorithm 1 achieves the regret bound

$$
\mathcal{R}\left(T, \mathcal{G}^{N}\right) \leq \frac{\Psi\left(A_{R}+1\right) \ln \left(\left(H_{S}+1\right) M\right)}{\eta}+\frac{M T \eta}{2}
$$

where $\Psi$ is an upper bound on the cardinality of the child node groups $\phi$, i.e., $\Psi \geq|\phi|$ for all $\phi, H_{S}$ is an upper bound on the cardinality of $\Phi_{i}$, i.e., $H_{S} \geq\left|\Phi_{i}\right|$ for all $i$, and $A_{R}$ is an upper bound on the minimum number of splittings needed in the hierarchical structure to model the optimal partition with $R$ disjoint regions.

Proof of Theorem 2: If the optimal expert is defined over the root node, i.e., $A_{R}=0$, its prior weight in the mixture is

$$
\beta_{1, j}=\frac{1}{\left(\left|\Phi_{i}\right|+1\right) M} \geq \frac{1}{\left(H_{S}+1\right) M} .
$$

With each split in the hierarchical structure (i.e., with each move down the hierarchy), the prior weights of the experts are divided by a factor that is at most $\left(H_{S}+1\right)^{\Psi} M^{\Psi-1}$. Thus, in case we need $A_{R}$ splittings to model the partition corresponding to the optimal expert, its prior weight is

$$
\beta_{1, j} \geq\left(H_{S}+1\right)^{-A_{R} \Psi-1} M^{A_{R}-A_{R} \Psi-1} .
$$

Since $A_{R} \geq 1$ and $\Psi \geq 1$, we have

$$
\beta_{1, j} \geq\left(H_{S}+1\right)^{-\Psi\left(A_{R}+1\right)} M^{-\Psi\left(A_{R}+1\right)} .
$$

Hence,

$$
\ln \left(1 / \beta_{1, j}\right) \leq \Psi\left(A_{R}+1\right) \ln \left(\left(H_{S}+1\right) M\right) .
$$

Substituting (20) into (6) concludes the proof.

Corollary 1: By setting

$$
\eta=\sqrt{\frac{2 \Psi\left(A_{R}+1\right) \ln \left(\left(H_{S}+1\right) M\right)}{M T}}
$$

we get the regret bound of

$$
\mathcal{R}\left(T, \mathcal{G}^{N}\right) \leq \sqrt{0.5 \Psi M T\left(A_{R}+1\right) \ln \left(\left(H_{S}+1\right) M\right)} .
$$

We next present several examples of hierarchical structures that can be employed by our algorithm with the introduced mathematical guarantees. Each structure has its own way of encoding the best arm selection policy, i.e., optimal arbitrary mapping. Hence, the proper selection of the hierarchical structure according to the target application leads to a smaller $A_{R}$ and a better performance, i.e., a regret upper bound vanishing faster in the average loss per round sense, together with the introduced weighting over the corresponding competition class $\mathcal{G}^{N}$ (see Section VI as well as the examples in the following).

\section{B. Example 1: Arbitrary Splitting}

If the hierarchical structure is an arbitrary splitting of $N$ leaf nodes into two groups, then $\Psi=2, H_{S}=2^{N-1}-1$, and $A_{R}=M-1$. Hence, the regret is upper bounded as

$$
\begin{aligned}
\mathcal{R}\left(T, \mathcal{G}^{N}\right) & \leq \frac{2 M \ln \left(2^{N-1} M\right)}{\eta}+\frac{M T \eta}{2} \\
& \leq \frac{2 M N \ln (M)}{\eta}+\frac{M T \eta}{2}
\end{aligned}
$$

where the last inequality uses $2 \leq M$.

\section{Example 2: Binary Tree}

In BTs, we have $\Psi=2$ and $H_{S}=1$. For a BT with $N$ leaf nodes, we need at most $\log _{2} N$ splitting to create each new region. Hence, $A_{R}=(R-1) \log _{2} N$. Therefore,

$$
\begin{aligned}
\mathcal{R}\left(T, \mathcal{G}^{N}\right) & \leq \frac{2\left((R-1) \log _{2} N+1\right) \ln (2 M)}{\eta}+\frac{M T \eta}{2} \\
& \leq \frac{2 R \log _{2} N \ln (2 M)}{\eta}+\frac{M T \eta}{2} .
\end{aligned}
$$

\section{Example 3: K-Ary Tree}

If the hierarchical structure is a $\mathrm{K}$-ary tree (for $K=2$, this becomes a BT) with $N$ leaf nodes and depth $D=\log _{K} N$, then $\Psi=K, H_{S}=1$, and $A_{R}=(R-1) \log _{K} N$. Therefore, we have

$$
\begin{aligned}
\mathcal{R}\left(T, \mathcal{G}^{N}\right) & \leq \frac{K\left(1+(R-1) \log _{K} N\right) \ln (2 M)}{\eta}+\frac{M T \eta}{2} \\
& \leq \frac{K R \log _{K} N \ln (2 M)}{\eta}+\frac{M T \eta}{2} .
\end{aligned}
$$




\section{E. Example 4: Lexicographical Splitting Graph}

In a lexicographal splitting graph with $N$ leaf nodes, we have $\Psi=2, H_{S}=N-1$, and $A_{R}=R-1$. Hence,

$$
\mathcal{R}\left(T, \mathcal{G}^{N}\right) \leq \frac{2 R \ln (N M)}{\eta}+\frac{M T \eta}{2} .
$$

\section{F. Example 5: K-Group Lexicographical Splitting}

If the hierarchical structure is a splitting of $N$ sequentially ordered leaf nodes into $K$ groups (when $K=2$, this structure becomes the lexicographical splitting graph), then $\Psi=K$, $H_{S}=\left(\begin{array}{c}N-1 \\ K-1\end{array}\right)$, and $A_{R}=\lceil(R-1) /(K-1)\rceil$. Therefore, the regret upper bound is

$$
\begin{aligned}
\mathcal{R}\left(T, \mathcal{G}^{N}\right) & \leq \frac{K\left(\left\lceil\frac{R-1}{K-1}\right\rceil+1\right) \ln \left(\left(1+\left(\begin{array}{c}
N-1 \\
K-1
\end{array}\right)\right) M\right)}{\eta}+\frac{M T \eta}{2} \\
& \leq \frac{K(R+2 K) \ln (N M)}{\eta}+\frac{M T \eta}{2} .
\end{aligned}
$$

\section{G. Example 6: Arbitrary Position Splitting}

In this case, for a $d$-dimensional context space, we have $\Psi=2, H_{S}=d$, and $A_{R}=(R-1) \log _{2} N$. Therefore,

$$
\begin{aligned}
\mathcal{R}\left(T, \mathcal{G}^{N}\right) & \leq \frac{2\left((R-1) \log _{2} N+1\right) \ln ((d+1) M)}{\eta}+\frac{M T \eta}{2} \\
& \leq \frac{2 R \log _{2} N \ln ((d+1) M)}{\eta}+\frac{M T \eta}{2} .
\end{aligned}
$$

We have successfully achieved a regret bound of $O\left((M T R \ln N \ln M)^{1 / 2}\right)$ with proper selection of the learning rate. Note that typically $N \gg R$. Our regret bounds are only logarithmically dependent on $N$; hence, in soft- $O$ notation, we achieve the minimax optimal regret bound $\tilde{O}(\sqrt{T R})$.

Next and finally, we address the goal of achieving the performance of the best arm selection policy, i.e., the performance of the optimal arbitrary mapping (in the ultimate set $\mathcal{U}$ ) from the context space to the bandit arms, which is not necessarily in the competition class $\mathcal{G}^{N}$ but can be approximated arbitrarily well and almost perfectly, if desired, by the class by increasing $N$. The quantization process in our algorithm naturally produces an additive linear-in-time term in our regret against the truly optimal mapping in $\mathcal{U}$. In Section V, we assume that the arm losses are Lipschitz continuous in the context vectors at each specific round. With this assumption, we show that using a uniform quantization of the context space, and we can diminish the linear-in-time term in our regret against the optimal mapping in $\mathcal{U}$ by increasing the number of quantization levels $N$. Hence, we can achieve a performance as close as desired to the performance of the optimal mapping in $\mathcal{U}$.

\section{EFFicient Quantization Method to Asymptotically ACHIEVE the Optimal CONTEXT-BAsed ARM SELECTION}

Suppose that the context space is the $n$-dimensional space $S=[0,1]^{n}$. Using a hierarchical structure with $N$ leaf nodes, our quantization scheme is as follows. We split the context space into $2^{\left\lfloor\left(\log _{2} N\right) / n\right\rfloor+1}$ equal subspaces along the first $\log _{2} N(\bmod n)$ dimensions (of the total $n$ dimensions) and $2^{\left\lfloor\left(\log _{2} N\right) / n\right\rfloor}$ equal subspaces along the remaining dimensions.

Theorem 3: Using aforementioned quantization method for our algorithm, if the arm loss functions are the Lipschitz continuous with the Lipschitzness constant $c$, then the difference between the loss corresponding to the best mapping in $\mathcal{G}^{N}$ and the loss corresponding to the truly optimal mapping (in the ultimate $\operatorname{set}^{9} \mathcal{U}$ of all possible arbitrary mappings from the context space to the set of bandit arms) is upper bounded by

$$
\frac{2 c \sqrt{n}}{\sqrt[n]{N}}
$$

Proof of Theorem 3: Using this quantization method, the subspaces in the finest partition of the context space are $n$-dimensional cubes with the longest diagonal length equal to

$$
\sqrt{\frac{n-\left(\log _{2} N(\bmod n)\right)}{\left(2^{\left\lfloor\frac{\log _{2} N}{n}\right\rfloor}\right)^{2}}+\frac{\log _{2} N(\bmod n)}{\left(2^{\left\lfloor\frac{\log _{2} N}{n}+1\right\rfloor}\right)^{2}}} .
$$

Since $\log _{2} N(\bmod n) \geq 0$, this upper bound is at most equal to

$$
\sqrt{\frac{n}{2^{2\left\lfloor\frac{\log _{2} N}{n}\right\rfloor}}} \leq \frac{2 \sqrt{n}}{2^{\frac{\log _{2} N}{n}}}=\frac{2 \sqrt{n}}{\sqrt[n]{N}}
$$

Since the loss functions are Lipschitz continuous, the difference between the loss corresponding to the truly optimal mapping in $\mathcal{U}$ and the best mapping in $\mathcal{G}^{N}$ cannot exceed the Lipschitzness constant times the quantized cubes diagonal length, which concludes the proof.

Note that the Lipschitzness assumption does not intervene with the adversarial setting. The loss functions can be quite different in different rounds, and as long as they are Lipschitz continuous at each specific round, the assumption holds and our algorithm is competitive against the ultimate set of all possible arbitrary mappings $\mathcal{U}$. In this case, combining (29) with the regret bound in (22) directly concludes the following theorem.

Theorem 4: Consider a contextual $M$-armed bandit problem with the context space $S=[0,1]^{n}$, where the loss functions of the arms are the Lipschitz continuous with the constant $c$ at all rounds. If we use a hierarchical structure with $N$ leaf nodes following the quantization scheme described in Section V, the regret of Algorithm 1 against the truly optimal strategy in a $T$ round trial is upper bounded as follows:

$$
R(T, \mathcal{U}) \leq \sqrt{\frac{\Psi M T\left(A_{R}+1\right) \ln \left(\left(H_{S}+1\right) M\right)}{2}}+\frac{2 T c \sqrt{n}}{\sqrt[n]{N}}
$$

We emphasize that we can make the linear-in-time term of the upper bound in (32) as small as desired by growing the hierarchical structure and increasing the number of leaf nodes $N$, which is equal to the number of quantization levels.

\footnotetext{
${ }^{9}$ This ultimate set can be nonrigorously considered as $\mathcal{G}^{\infty}$.
} 


\section{EXPERIMENTS}

In this section, we demonstrate the performance of our algorithm in different scenarios involving both real and synthetic data. We demonstrate the performance of our main algorithm hierarchical structure-based bandits (HSB) with various hierarchical structures, including HSB-BT, lexicograph (HSB-LG), and arbitrary position splitting (HSB-APS) [33]. We compare the performance of our algorithm against the state-of-the-art adversarial bandit algorithms EXP3 and $S$-EXP3 [16]. In all of the experiments, the parameters of EXP3 and $S$-EXP3 algorithms are set to their optimal values according to their publication [16].

\section{A. Stationary Environment}

We first construct a game with 3-armed bandit, where the context space is the $1-\mathrm{D}$ space $S=[0,1]$. Each arm $i$ generates its loss according to the Bernoulli distribution with parameter $p_{i}$, i.e., the loss is equal to 1 with probability equal to $p_{i}$. These parameters, i.e., $p_{1}, p_{2}$, and $p_{3}$, depend on the context variable $s_{t}$ as

$$
\begin{aligned}
& p_{1}\left(s_{t}\right)=0.5+0.5 \sin \left(2 \pi s_{t}\right) \\
& p_{2}\left(s_{t}\right)=\sin \left(\pi s_{t}\right) \\
& p_{3}\left(s_{t}\right)=s_{t} .
\end{aligned}
$$

Here, the optimal strategy is defined as follows

$$
g\left(s_{t}\right)= \begin{cases}3, & s_{t}<0.5 \\ 1, & 0.5 \leq s_{t}<0.9182 \\ 2, & 0.9182 \leq s_{t}\end{cases}
$$

In this experiment, we generate the context variable $s_{t}$ randomly with uniform distribution over the context space, i.e., $[0,1]$, and compare the averaged cumulated loss performance, i.e., $\left(\sum_{\tau=1}^{t} l_{\tau, I_{\tau}}\right) / t$, for our algorithm $H S B$-BT with various depth parameters equal to 2, 5, and 10, S-EXP3 [16] with the same depth parameters, and EXP3 [16].

To this end, we generate 10 synthetic data sets of length $10^{5}$. To produce each data set, first, $10^{5}$ context variables $s_{t}$ are drawn according to a uniform probability distribution over the interval $[0,1]$. Then, the arm losses corresponding to different rounds are drawn from the Bernoulli distributions, parameters of which are determined according to (33). Each data set is presented to the algorithms 10 times, and the results are averaged. This process is repeated for all 10 data sets and the ensemble averages are plotted in Fig. 5. Two important results can be derived from the result of this experiment. First, our algorithm $H S B-B T$ outperforms both of the $S-E X P 3$ and EXP3 algorithms. Second, while increasing the depth uniformly improves the performance of our algorithm, it can degrade the performance of $S$-EXP3 due to the overtraining. The superior performance of our algorithm in this experiment is because of its fast convergence to the optimal mapping. Here, EXP3 has a fast convergence but it converges to a suboptimal mapping because it does not use the context information. On the other hand, S-EXP3 converges to the optimal mapping but needs a huge amount of data to get trained. Our algorithm uses an efficient adaptive combination of the experts with intelligent

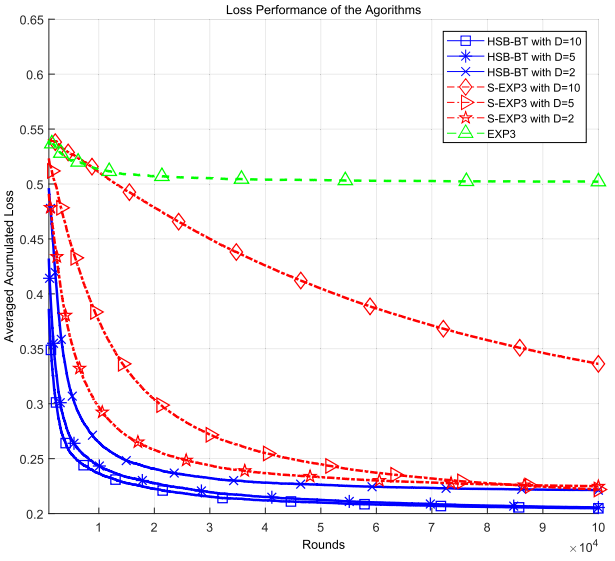

Fig. 5. Averaged accumulated loss of HSB-BT, S-EXP3, and EXP3 on the data sets defined using (33).

initial weights to obtain the advantages of both EXP3 and $S$-EXP3 algorithms while mitigating their disadvantages.

\section{B. Nonstationary Environment}

In this part, we illustrate the averaged cumulated loss performance of the algorithms in a nonstationary environment. To this end, we construct 10 different data sets of length $10^{5}$ as in Section VI-A. However, here, the arm losses follow a model as in (33) in the first quarter of the rounds and the following model in the rest of the rounds:

$$
\begin{aligned}
& p_{1}\left(s_{t}\right)=\sin \left(\pi s_{t}\right) \\
& p_{2}\left(s_{t}\right)=s_{t} \\
& p_{3}\left(s_{t}\right)=0.5+0.5 \sin \left(2 \pi s_{t}\right) .
\end{aligned}
$$

Hence, we have an abrupt change in the model of the arms within the rounds. Each data set is presented to the algorithms 10 times and the results are averaged. This process is repeated for all 10 data sets and the ensemble averages are plotted in Fig. 6. As shown in Fig. 6, our algorithm HSB-BT not only outperforms its competitor before the rapid change in the model of the bandit arms but also adopts better to this rapid change in comparison to the competitors.

\section{Real-Life Online Advertisement Data Set}

In this section, we demonstrate the superior performance of our algorithms $H S B-B T$ and $H S B-L G$ against their natural competitors $E X P 3$ and $S$-EXP3 over the well-known real-life data set provided by Yahoo! Research. This data set contains a user click log for news articles displayed in the featured tab of the Today Module on Yahoo!'s front page, within October 2 to 16, 2011. The data set contains 28041015 user visits. For each visit, the user is associated with a binary feature vector of dimension 136 that contains information about the user, such as age, gender, behavior targeting features, and so on. We used an unbiased off-line evaluation method as in [48] to test the competitors over this data set. A brief pseudocode of this evaluation method is shown in Algorithm 2. In this experiment, we ran a principal component analysis algorithm [49] over the first 5\% of the data to get the principal components of the feature vectors. We mapped the feature 


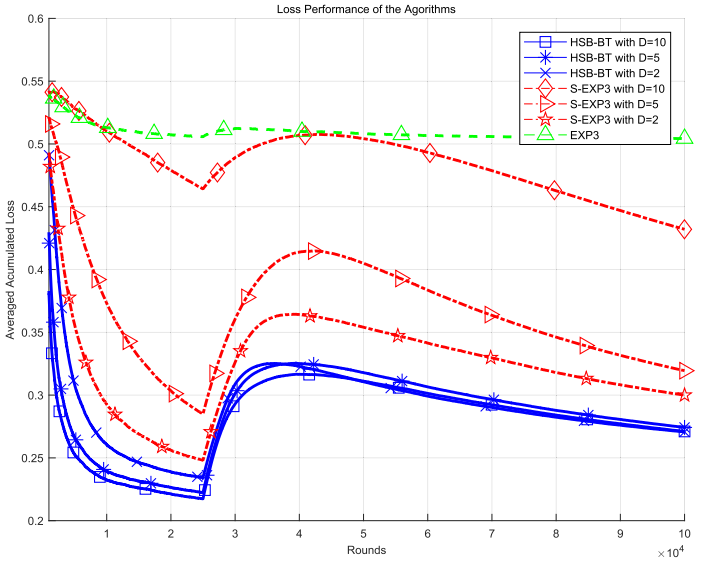

Fig. 6. Averaged accumulated loss of $H S B-B T, S-E X P 3$, and EXP3 on the data sets, as described in Section VI-B, involving a rapid change in the behavior of the arms after $25 \%$ of the rounds.

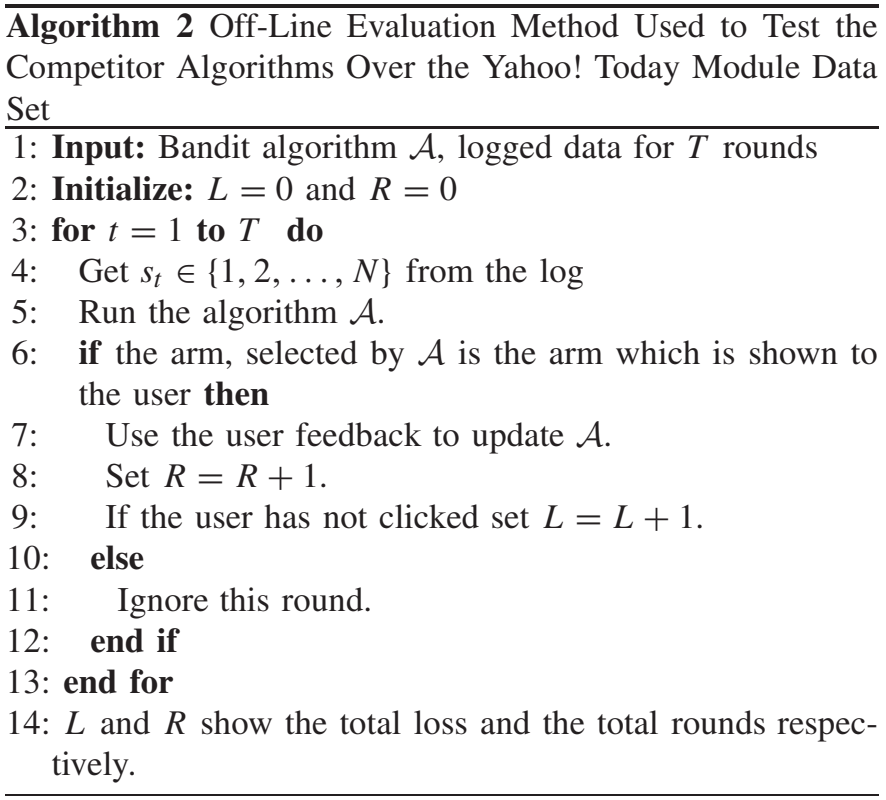

vectors over the first principal component to form a set of 1-D context variables. We used these context variables for $S$-EXP3, $H S B-B T$, and $H S B-L G$ algorithms. We tested the EXP3 and $S$ EXP3 algorithms with several depth parameters, while their parameters were set to their optimum values [16]. However, since we do not have any information about the number of disjoint regions in the optimal mapping, i.e., $R$, the $\eta$ parameter for the $H S B-B T$ and $H S B-L G$ algorithms cannot be tuned to the optimum value analytically. In this experiment, in order to have a fair comparison, we set the $\eta$ parameter of the $H S B-B T$ and $H S B-L G$ algorithm with a specific depth equal to the $\eta$ parameter of the $S$-EXP3 algorithm with the same depth. We emphasize that no numerical optimization is done for the $\eta$ parameter of our algorithms. The percentage of user clicks for different algorithms is shown in Fig. 7. As shown in Fig. 7, our algorithms outperform both of the S-EXP3 and EXP3 algorithms even though the learning rate parameters of our

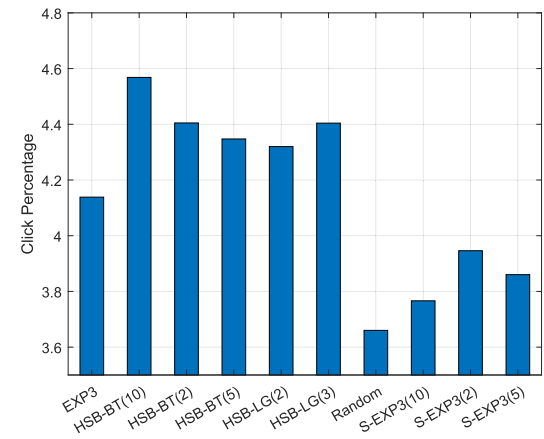

Fig. 7. Percentage of click in the Yahoo! Today Module data set.

algorithms are not tuned to the optimum values due to the lack of knowledge on the parameter $R$.

\section{Real-Life Classification Data Set}

In this experiment, we use the well-known Landsat data set [50] to show how our algorithm can be employed for online multiclass classification in the error-correcting output codes (ECOCs) framework [51]. This data set consists of 6435 samples from six classes. The feature vectors are 36-D integer vectors.

In the ECOC framework, given a set of $C$ classes, we assign a binary code word of length $N_{C}$ to each one of the classes. We arrange these code words as rows of a coding matrix $M_{C} \in\{+1,-1\}^{C \times N_{C}}$. We consider each one of the $N_{C}$ columns of $M_{C}$ as a binary classification problem and run a binary classifier over each column. The $i$ th classifier is to learn whether the $i$ th bit of the code word is +1 or -1 . In order to label a new sample, the feature vector is fed to the binary classifiers to obtain a code word based on their outputs. We then decide on the label of the sample based on its code word.

In this experiment, we use the one-versus-all coding [51] to form our coding matrix and run six Online Perceptrons in parallel as our binary classifiers. We use the code words obtained from the Perceptrons as our context vectors and the classes as our bandit arms. We provide our algorithm HSB with the context vectors and label the sample based on the arm suggested by the algorithm. Then, we observe the true label and suffer a loss equal to 1 in case of incorrect label. The competitors in this experiment are our algorithm HSB with two different hierarchical structures of "Arbitrary Position Splitting" (HSB-APS) and "Binary Tree" (HSB-BT), alongside EXP3, S-EXP3, and Hamming Decoding [51]. The learning parameters of the algorithms are set to their optimal value.

We emphasize that while the Hamming Decoder knows the code words corresponding the classes a priori, other competitors do not use this information and try to learn the best mapping from the context space, i.e., code words space, to the classes. For presentation simplicity, we have splitted the samples into nine consecutive epochs and averaged the number of errors over each epoch. As shown in Fig. 8, the algorithms $S$-EXP3, $H S B-B T$, and $H S B$ - $A P S$ compensate their lack of information on the coding matrix (compared to 


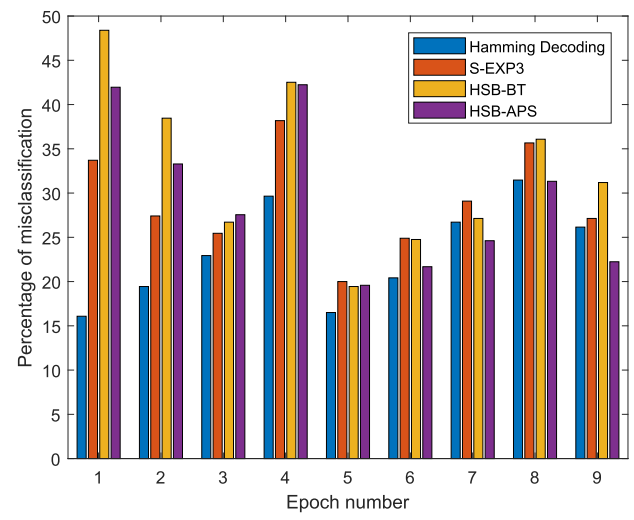

Fig. 8. Percentage of misclassification of the competitors over nine consecutive epochs of length 715 .

the Hamming Decoder) as time goes on. Among them, HSB-APS outperforms the others and even Hamming Decoder in the last three epochs as expected.

\section{CONCLUSION}

We studied the contextual multiarmed bandit problem in an adversarial setting and introduced a truly online and low-complexity algorithm that asymptotically achieves the performance of the best context-dependent bandit arm selection policy. Our core algorithm quantizes the space of the context vectors into a large number of disjoint regions using an efficient quantization method and forms the class of all mappings from these regions to the bandit arms. Then, it adaptively combines these mappings in a mixture-of-experts setting and achieves the performance of the best mapping in the class. We prove performance upper bounds for the introduced algorithm. These upper bounds show that we achieve the performance of the truly optimal mapping (which might be out of our class of mappings) by increasing the number of quantization levels. We use hierarchical structures to implement our algorithm in an efficient way, such that the computational complexity is log-linear in the number of quantization levels. We have no statistical assumptions on the behavior of the context vectors and the bandit arms, and hence, our results are guaranteed to hold in an individual sequence manner. Through extensive set of experiments involving synthetic and real data, we demonstrate the significant performance gains achieved by the proposed algorithm in comparison to the state-of-the-art techniques.

\section{APPENDIX A \\ PROOF OF THEOREM 1}

From the definition, denoting the mapping followed by the $j$ th expert by $g_{j}($.$) , we have:$

$$
\mathcal{R}\left(T, E_{j}\right)=\mathbb{E}\left[\sum_{t=1}^{T} l_{t, I_{t}}-\sum_{t=1}^{T} l_{t, g_{j}\left(\mathbf{s}_{t}\right)}\right]
$$

where $l_{t, I_{t}}$ can be expanded as

$$
\begin{aligned}
l_{t, I_{t}}= & \mathbb{E}_{j \sim \boldsymbol{\beta}_{t}} \tilde{l}_{t, g_{j}\left(\mathbf{s}_{t}\right)} \\
= & \frac{1}{\eta}\left(\ln \left(\mathbb{E}_{j \sim \boldsymbol{\beta}_{t}} e^{-\eta \tilde{l}_{t, g_{j}}\left(\mathbf{S}_{t}\right)}\right)+\eta \mathbb{E}_{j \sim \boldsymbol{\beta}_{t}} \tilde{l}_{t, g_{j}\left(\mathbf{S}_{t}\right)}\right) \\
& -\frac{1}{\eta} \ln \mathbb{E}_{j \sim \boldsymbol{\beta}_{t}} e^{-\eta \tilde{l}_{t, g_{j}\left(\mathbf{S}_{t}\right)}} .
\end{aligned}
$$

The first term in (37) can be bounded using the inequalities $\ln x \leq x-1$ and $\exp (-x)-1+x \leq x^{2} / 2$ for all $x \geq 0$, as

$$
\begin{aligned}
& \ln \left(\mathbb{E}_{j \sim \boldsymbol{\beta}_{t}} e^{-\eta \tilde{l}_{t, g_{j}}\left(\mathbf{S}_{t}\right)}\right)+\eta \mathbb{E}_{j \sim \boldsymbol{\beta}_{t}} \tilde{l}_{t, g_{j}\left(\mathbf{S}_{t}\right)} \\
& \leq \mathbb{E}_{j \sim \boldsymbol{\beta}_{t}}\left[e^{-\eta \tilde{l}_{t, g_{j}}\left(\mathbf{S}_{t}\right)}-1+\eta \tilde{l}_{t, g_{j}\left(\mathbf{s}_{t}\right)}\right] \\
& \leq \mathbb{E}_{j \sim \boldsymbol{\beta}_{t}} \frac{\eta^{2} \tilde{l}_{t, g_{j}}^{2}\left(\mathbf{S}_{t}\right)}{2}=\frac{\eta^{2} l_{t, I_{t}}^{2}}{2 p_{t, I_{t}}} \leq \frac{\eta^{2}}{2 p_{t, I_{t}}}
\end{aligned}
$$

In order to bound the second term in (37), we just rewrite the expectation using (4) as follows. For $t=1$, we have

$$
-\frac{1}{\eta} \ln \mathbb{E}_{j \sim \beta_{1}} e^{-\eta \tilde{l}_{1, g_{j}}\left(\mathbf{S}_{1}\right)}=-\frac{1}{\eta} \ln \frac{\sum_{j=1}^{M^{N}} \alpha_{1, j} e^{-\eta \tilde{l}_{1, g_{j}}\left(\mathbf{S}_{1}\right)}}{\sum_{j=1}^{M^{N}} \alpha_{1, j}}
$$

and for $t \geq 2$, we have

$$
-\frac{1}{\eta} \ln \mathbb{E}_{j \sim \beta_{t}} e^{-\eta \tilde{l}_{t, g_{j}}\left(\mathbf{S}_{t}\right)}=-\frac{1}{\eta} \ln \frac{\sum_{j=1}^{M^{N}} \alpha_{1, j} e^{-\eta \sum_{\tau=1}^{t} \tilde{l}_{\tau, g_{j}}\left(\mathbf{S}_{\tau}\right)}}{\sum_{j=1}^{M^{N}} \alpha_{1, j} e^{-\eta \sum_{\tau=1}^{t-1} \tilde{l}_{\tau, g_{j}\left(\mathbf{S}_{\tau}\right)}}} .
$$

Putting the bounds in (38) and (40) into (37), we have

$$
\begin{aligned}
\sum_{t=1}^{T} l_{t, I_{t}} \leq-\frac{1}{\eta}\left(\sum_{t=2}^{T} \ln \frac{\sum_{j=1}^{M^{N}} \alpha_{1, j} e^{-\eta \sum_{\tau=1}^{t} \tilde{l}_{\tau, g_{j}}\left(\mathbf{S}_{\tau}\right)}}{\sum_{j=1}^{M^{N}} \alpha_{1, j} e^{-\eta \sum_{\tau=1}^{t-1} \tilde{l}_{\tau, g_{j}\left(\mathbf{S}_{\tau}\right)}}}\right. \\
\left.+\ln \frac{\sum_{j=1}^{M^{N}} \alpha_{1, j} e^{-\eta \tilde{l}_{1, g_{j}\left(\mathbf{S}_{1}\right)}}}{\sum_{j=1}^{M^{N}} \alpha_{1, j}}\right)+\frac{\eta T}{2 p_{t, I_{t}}}
\end{aligned}
$$

Opening the first two terms in (41), we have

$$
\begin{aligned}
\sum_{t=1}^{T} l_{t, I_{t}} \leq-\frac{1}{\eta} \ln \sum_{j=1}^{M^{N}} \alpha_{1, j} e^{-\eta \sum_{\tau=1}^{T} \tilde{l}_{\tau, g_{j}}\left(\mathbf{S}_{\tau}\right)} & \\
& +\frac{1}{\eta} \ln \sum_{j=1}^{M^{N}} \alpha_{1, j}+\frac{\eta T}{2 p_{t, I_{t}}}
\end{aligned}
$$

Since $\sum_{j=1}^{M^{N}} \alpha_{1, j} e^{-\eta \sum_{\tau=1}^{T} \tilde{l}_{\tau, g_{j}}\left(\mathbf{S}_{\tau}\right)} \leq \alpha_{1, j} e^{-\eta \sum_{\tau=1}^{T} \tilde{l}_{\tau, g_{j}}\left(\mathbf{S}_{\tau}\right)}$, we have

$$
\begin{aligned}
\sum_{t=1}^{T} l_{t, I_{t}} & \leq-\frac{1}{\eta} \ln \alpha_{1, j}+\sum_{\tau=1}^{T} \tilde{l}_{\tau, g_{j}\left(\mathbf{s}_{\tau}\right)}+\frac{1}{\eta} \ln \sum_{j=1}^{M^{N}} \alpha_{1, j}+\frac{\eta T}{2 p_{t, I_{t}}} \\
& =\frac{\ln 1 / \beta_{1, j}}{\eta}+\frac{\eta T}{2 p_{t, I_{t}}}+\sum_{\tau=1}^{T} \tilde{l}_{\tau, g_{j}\left(\mathbf{s}_{\tau}\right) .}
\end{aligned}
$$

Taking expectation from both sides (with respect to $I_{t} \sim \mathbf{p}_{t}$ )

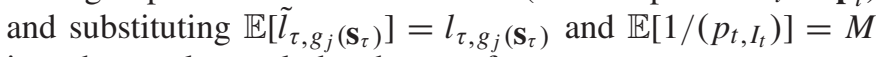
into the result concludes the proof.

\section{APPENDIX B}

\section{PROOF OF PROPOSITION 1}

We prove this proposition using induction. For leaf nodes where $\Phi_{i}=\emptyset$, we have

$$
w_{t, i}=\frac{1}{M} \sum_{m=1}^{M} \alpha_{t, m, i}
$$


From the definition of $\alpha_{t, m, i}$ in (7), we have

$$
w_{t, i}=\sum_{m=1}^{M} \frac{1}{M} \exp \left(-\eta \sum_{\substack{\tau<t \\ \mathbf{s}_{\tau} \in r_{i}}} \tilde{l}_{\tau, m}\right)=\sum_{k \in \Gamma_{i}} \alpha_{t, k}
$$

where $\alpha_{1, k}=1 / M$ for all $k \in \Gamma_{i}$.

Consider the node $v_{i}$. Suppose $\forall \phi \in \Phi_{i}, \forall j \in \phi$, we have

$$
w_{t, j}=\sum_{k \in \Gamma_{j}} \alpha_{t, k}
$$

It suffices to show that

$$
w_{t, i}=\sum_{k \in \Gamma_{i}} \alpha_{t, k}
$$

The set of experts defined over $v_{i}$, i.e., $\Gamma_{i}$, can be decomposed into the following subsets.

1) $\Gamma_{i}^{o}$ : The set of experts, which map the whole context space into a fixed arm. This set contains $M$ experts.

2) $\Gamma_{i}^{\phi}, \phi \in \Phi_{i}$ : The set of experts, which partition the context space into the regions $r_{j}, j \in \phi$, and follow a specific expert over each node $j \in \phi$, based on the observed $\mathbf{s}_{t}$. If $\mathbf{s}_{t} \in r_{j}$, the experts in $\Gamma_{i}^{\phi}$ follow the experts in $\Gamma_{j}$. This set contains $\prod_{j \in \phi}\left|\Gamma_{j}\right|$ experts. Each experts in $\Gamma_{i}^{\phi}$ can be represented by a vector of experts $\mathbf{k}_{\phi} \in \prod_{j \in \phi} \Gamma_{j}$, where $\mathbf{k}_{\phi}(j)$ is an expert defined over node $j$.

We emphasize that even though we have

$$
\Gamma_{i}^{o} \cup\left(\bigcup_{\phi \in \Phi_{i}} \Gamma_{i}^{\phi}\right)=\Gamma_{i}
$$

the intersection of any two of these $\left|\Phi_{i}\right|+1$ subsets is not empty necessarily. In particular, the $M$ experts in $\Gamma_{i}^{o}$ are also included among the elements of $\Gamma_{i}^{\phi}$ for all $\phi \in \Phi_{i}$. In fact, each expert in $\Gamma_{i}^{o}$ can be seen as an expert that partitions the context space into $r_{j}$ s for $j \in \phi$ and follows the experts that select a fixed arm $m$ over all the nodes $v_{j}$ s.

We have

$$
\prod_{j \in \phi} w_{t, j}=\prod_{j \in \phi}\left(\sum_{k \in \Gamma_{j}} \alpha_{t, k}\right)=\sum_{\mathbf{k}_{\phi} \in \prod_{j \in \phi} \Gamma_{j}}\left(\prod_{j} \alpha_{t, \mathbf{k}_{\phi}(j)}\right) .
$$

We open the product term as

$$
\begin{aligned}
\prod_{j} & \alpha_{t, \mathbf{k}_{\phi}(j)} \\
& =\prod_{j} \alpha_{1, \mathbf{k}_{\phi}(j)} \exp \left(-\eta \sum_{\tau<t} \sum_{j} \tilde{l}_{\tau, g_{\mathbf{k}_{\phi}(j)}}\left(\mathbf{S}_{\tau}\right) \mathbf{1}_{\left\{\mathbf{s}_{\tau} \in r_{j}\right\}}\right) \\
& =\prod_{j} \alpha_{1, \mathbf{k}_{\phi}(j)} \exp \left(-\eta \sum_{\substack{\tau<t \\
\mathbf{s}_{\tau} \in r_{i}}} \tilde{l}_{\tau, g_{\mathbf{k}_{\phi}}}\left(\mathbf{s}_{\tau}\right)\right) .
\end{aligned}
$$

Substituting (50) into (9), we get

$$
\begin{aligned}
w_{t, i}= & \frac{1}{\left(\left|\Phi_{i}\right|+1\right) M} \sum_{k \in \Gamma_{i}^{o}} \alpha_{t, k}+\frac{1}{\left|\Phi_{i}\right|+1} \\
& \times \sum_{\phi \in \Phi_{i}}\left(\sum _ { \mathbf { k } _ { \phi } \in \prod _ { j \in \phi } \Gamma _ { j } } \alpha _ { 1 , \mathbf { k } _ { \phi } } \operatorname { e x p } \left(-\eta \sum_{\substack{\tau<t \\
\mathbf{s}_{\tau} \in r_{i}}} \tilde{l}_{\left.\left.\tau, g_{\mathbf{k}_{\phi}\left(\mathbf{s}_{\tau}\right)}\right)\right)}^{=}\right.\right. \\
= & \frac{1}{\left(\left|\Phi_{i}\right|+1\right) M} \sum_{k \in \Gamma_{i}^{o}} \alpha_{t, k}+\frac{1}{\left(\left|\Phi_{i}\right|+1\right)} \sum_{\phi \in \Phi_{i}} \sum_{k \in \Gamma_{i}^{\phi}} \alpha_{t, k} \\
= & \sum_{k \in \Gamma_{i}} \alpha_{t, k}
\end{aligned}
$$

where

$$
\begin{aligned}
& \alpha_{1, k}=\frac{1}{\left(\left|\Phi_{i}\right|+1\right) M} \mathbf{1}_{\left\{k \in \Gamma_{i}^{o}\right\}} \\
& \quad+\frac{1}{\left|\Phi_{i}\right|+1} \sum_{\phi \in \Phi_{i}}\left(\mathbf{1}_{\left\{k=\mathbf{k}_{\phi}\right\}} \prod_{j \in \phi} \alpha_{1, \mathbf{k}_{\phi}(j)}\right) .
\end{aligned}
$$

\section{APPENDIX C}

\section{PROOF OF PROPOSITION 2}

Consider a specific bandit arm $m^{*}$. Given the context vector $\mathbf{s}_{t}$, for all $m \in\{1,2, \ldots, M\}$, and for all nodes $v_{i}$ in the hierarchy, we define the variables $\tilde{\alpha}_{t, m, i}$ as

$$
\tilde{\alpha}_{t, m, i}= \begin{cases}0, & \mathbf{s}_{t} \in r_{i}, \quad m \neq m^{*} \\ \alpha_{t, m, i}, & \text { otherwise. }\end{cases}
$$

Now, from the definition of $\gamma_{t, m, i}$ in (13), we have

$$
\begin{aligned}
\gamma_{t, m^{*}, i}=\frac{1}{\left(\left|\Phi_{i}\right|+1\right) M} \sum_{m=1}^{M} \tilde{\alpha}_{t, m, i} & \\
& \quad+\frac{1}{\left(\left|\Phi_{i}\right|+1\right)} \sum_{\phi \in \Phi_{i}}\left(\prod_{j \in \phi} \tilde{w}_{t, j}\right) .
\end{aligned}
$$

The exact same lines of the Proof of Theorem 1 hold to show that

$$
\tilde{w}_{t, i}=\sum_{k \in \Gamma_{i}} \tilde{\alpha}_{t, k}
$$

where

$$
\tilde{\alpha}_{t, k}= \begin{cases}\alpha_{t, k}, & g_{k}\left(\mathbf{s}_{t}\right)=m^{*} \\ 0, & \text { otherwise. }\end{cases}
$$

Hence, (15) holds.

\section{REFERENCES}

[1] J. Lin and D.-X. Zhou, "Online learning algorithms can converge comparably fast as batch learning," IEEE Trans. Neural Netw. Learn. Syst., vol. 29, no. 6, pp. 2367-2378, Jun. 2018.

[2] L. Jian, S. Shen, J. Li, X. Liang, and L. Li, "Budget online learning algorithm for least squares SVM," IEEE Trans. Neural Netw. Learn. Syst., vol. 28, no. 9, pp. 2076-2087, Sep. 2017.

[3] A. Rakotomamonjy, S. Koço, and L. Ralaivola, "Greedy methods, randomization approaches, and multiarm bandit algorithms for efficient sparsity-constrained optimization," IEEE Trans. Neural Netw. Learn. Syst., vol. 28, no. 11, pp. 2789-2802, Nov. 2017. 
[4] J. Peng, A. J. Aved, G. Seetharaman, and K. Palaniappan, "Multiview boosting with information propagation for classification," IEEE Trans. Neural Netw. Learn. Syst., vol. 29, no. 3, pp. 657-669, Mar. 2017.

[5] G. Ditzler, R. Polikar, and G. Rosen, "A sequential learning approach for scaling up filter-based feature subset selection," IEEE Trans. Neural Netw. Learn. Syst., vol. 29, no. 6, pp. 2530-2544, Jun. 2018.

[6] R. J. Meyer and Y. Shi, "Sequential choice under ambiguity: Intuitive solutions to the armed-bandit problem," Manage. Sci., vol. 41, no. 5, pp. 817-834, 1995 .

[7] S. Shalev-Shwartz, "Online learning and online convex optimization," Found. Trends Mach. Learn., vol. 4, no. 2, pp. 107-194, 2012.

[8] N. Cesa-Bianchi and G. Lugosi, "Combinatorial bandits," J. Comput. Syst. Sci., vol. 78, no. 5, pp. 1404-1422, Sep. 2012.

[9] S. E. Yuksel, J. N. Wilson, and P. D. Gader, "Twenty years of mixture of experts," IEEE Trans. Neural Netw. Learn. Syst., vol. 23, no. 8, pp. 1177-1193, Aug. 2012.

[10] H. Ozkan, M. A. Donmez, S. Tunc, and S. S. Kozat, "A deterministic analysis of an online convex mixture of experts algorithm," IEEE Trans. Neural Netw. Learn. Syst., vol. 26, no. 7, pp. 1575-1580, Jul. 2015.

[11] A. J. Bean and A. C. Singer, "Universal switching and side information portfolios under transaction costs using factor graphs," IEEE J. Sel. Topics Signal Process., vol. 6, no. 4, pp. 351-365, Aug. 2012.

[12] A. C. Singer, S. S. Kozat, and M. Feder, "Universal linear least squares prediction: Upper and lower bounds," IEEE Trans. Inf. Theory, vol. 48, no. 8, pp. 2354-2362, Aug. 2002.

[13] A. C. Singer and M. Feder, "Universal linear prediction by model order weighting," IEEE Trans. Signal Process., vol. 47, no. 10, pp. 2685-2699, Oct. 1999.

[14] T. Moon and T. Weissman, "Universal FIR MMSE filtering," IEEE Trans. Signal Process., vol. 57, no. 3, pp. 1068-1083, Mar. 2009.

[15] T. Mannucci, E. J. van Kampen, C. de Visser, and Q. Chu, "Safe exploration algorithms for reinforcement learning controllers," IEEE Trans. Neural Netw. Learn. Syst., vol. 29, no. 4, pp. 1069-1081, Apr. 2018.

[16] S. Bubeck et al., "Regret analysis of stochastic and nonstochastic multiarmed bandit problems," Found. Trends Mach. Learn., vol. 5, no. 1, pp. 1-122, 2012.

[17] L. Li, W. Chu, J. Langford, and R. E. Schapire, "A contextual-bandit approach to personalized news article recommendation," in Proc. 19th Int. Conf. World Wide Web (WWW). New York, NY, USA: ACM, 2010, pp. 661-670.

[18] C. Tekin, S. Zhang, and M. van der Schaar, "Distributed online learning in social recommender systems," IEEE J. Sel. Topics Signal Process., vol. 8, no. 4, pp. 638-652, Aug. 2014.

[19] L. Tang, Y. Jiang, L. Li, and T. Li, "Ensemble contextual bandits for personalized recommendation," in Proc. 8th ACM Conf. Recommender Syst. (RecSys). New York, NY, USA: ACM, 2014, pp. 73-80.

[20] X. Luo, M. Zhou, S. Li, Z. You, Y. Xia, and Q.-S. Zhu, "A nonnegative latent factor model for large-scale sparse matrices in recommender systems via alternating direction method," IEEE Trans. Neural Netw. Learn. Syst., vol. 27, no. 3, pp. 579-592, Mar. 2016.

[21] J. P. Hardwick and Q. F. Stout, "Bandit strategies for ethical sequential allocation," Comput. Sci. Statist., vol. 23, pp. 421-424, 1991.

[22] Y. Gai, B. Krishnamachari, and R. Jain, "Learning multiuser channel allocations in cognitive radio networks: A combinatorial multi-armed bandit formulation," in Proc. IEEE Symp. New Frontiers Dyn. Spect., Apr. 2010, pp. 1-9.

[23] L. Lai, H. Jiang, and H. V. Poor, "Medium access in cognitive radio networks: A competitive multi-armed bandit framework," in Proc. 42nd Asilomar Conf. Signals, Syst. Comput., Oct. 2008, pp. 98-102.

[24] T. Lu, D. Pál, and M. Pál, "Contextual multi-armed bandits," in Proc. AISTATS, 2010, pp. 485-492.

[25] T. L. Lai, P. W. Lavori, and K. W. Tsang, "Adaptive design of confirmatory trials: Advances and challenges," Contemp. Clin. Trials, vol. 45, pp. 93-102, Nov. 2015.

[26] K. Liu and Q. Zhao, "Distributed learning in multi-armed bandit with multiple players," IEEE Trans. Signal Process., vol. 58, no. 11, pp. 5667-5681, Nov. 2010.

[27] M. Tokic, "Adaptive $\epsilon$-greedy exploration in reinforcement learning based on value differences," in KI: Advances in Artificial Intelligence. Berlin, Germany: Springer, 2010, pp. 203-210.

[28] O. Chapelle and L. Li, "An empirical evaluation of thompson sampling," in Proc. Adv. Neural Inf. Process. Syst., 2011, pp. 2249-2257.

[29] J. Langford and T. Zhang, "The Epoch-Greedy algorithm for multiarmed bandits with side information," in Proc. Adv. Neural Inf. Process. Syst., 2008, pp. 817-824.
[30] S. R. Bulò, B. Biggio, I. Pillai, M. Pelillo, and F. Roli, "Randomized prediction games for adversarial machine learning," IEEE Trans. Neural Netw. Learn. Syst., vol. 28, no. 11, pp. 2466-2478, Nov. 2017.

[31] L. Tang, R. Rosales, A. Singh, and D. Agarwal, "Automatic ad format selection via contextual bandits," in Proc. 22nd ACM Int. Conf. Conf. Inf. Knowl. Manage. (CIKM). New York, NY, USA: ACM, 2013, pp. 1587-1594

[32] P. Auer, N. Cesa-bianchi, Y. Freund, and R. E. Schapire, "The nonstochastic multiarmed bandit problem," SIAM J. Comput., vol. 32, no. 1, pp. 48-77, 2002.

[33] F. M. J. Willems, Y. M. Shtarkov, and T. J. Tjalkens, "Context weighting for general finite-context sources," IEEE Trans. Inf. Theory, vol. 42, no. 5, pp. 1514-1520, Sep. 1996.

[34] A. Agarwal, D. Hsu, S. Kale, J. Langford, L. Li, and R. Schapire, "Taming the monster: A fast and simple algorithm for contextual bandits," in Proc. Int. Conf. Mach. Learn., 2014, pp. 1638-1646.

[35] M. Dudik et al., "Efficient optimal learning for contextual bandits," in Proc. 27th Conf. Annu. Conf. Uncertainty Artif. Intell. (UAI). Corvallis, OR, USA: AUAI Press, 2011, pp. 169-178.

[36] P. Auer, "Using confidence bounds for exploitation-exploration tradeoffs," J. Mach. Learn. Res., vol. 3, pp. 397-422, Nov. 2003.

[37] A. Agarwal, M. Dudík, S. Kale, J. Langford, and R. E. Schapire, "Contextual bandit learning with predictable rewards," in Proc. AISTATS, 2012, pp. 19-26.

[38] V. Syrgkanis, A. Krishnamurthy, and R. E. Schapire, "Efficient algorithms for adversarial contextual learning," in Proc. 33rd Int. Conf. Mach. Learn., vol. 48, M. F. Balcan and K. Q. Weinberger, Eds. New York, NY, USA: PMLR, Jun. 2016, pp. 2159-2168.

[39] F. M. J. Willems, Y. M. Shtarkov, and T. J. Tjalkens, "The context-tree weighting method: Basic properties," IEEE Trans. Inf. Theory, vol. 41, no. 3, pp. 653-664, May 1995.

[40] K. Sadakane, T. Okazaki, and H. Imai, "Implementing the context tree weighting method for text compression," in Proc. Data Compress. Conf. (DCC), Mar. 2000, pp. 123-132.

[41] I. Csiszár and Z. Talata, "Context tree estimation for not necessarily finite memory processes, via BIC and MDL," IEEE Trans. Inf. Theory, vol. 52, no. 3, pp. 1007-1016, Mar. 2006.

[42] T. Dumont, "Context tree estimation in variable length hidden Markov models," IEEE Trans. Inf. Theory, vol. 60, no. 6, pp. 3196-3208, Jun. 2014.

[43] F. Babich, O. E. Kelly, and G. Lombardi, "A context-tree based model for quantized fading," IEEE Commun. Lett., vol. 3, no. 2, pp. 46-48, Feb. 1999.

[44] S. S. Kozat, A. C. Singer, and G. C. Zeitler, "Universal piecewise linear prediction via context trees," IEEE Trans. Signal Process., vol. 55, no. 7, pp. 3730-3745, Jul. 2007.

[45] N. D. Vanli and S. S. Kozat, "A comprehensive approach to universal piecewise nonlinear regression based on trees," IEEE Trans. Signal Process., vol. 62, no. 20, pp. 5471-5486, Oct. 2014.

[46] H. Ozkan, N. D. Vanli, and S. S. Kozat, "Online classification via selforganizing space partitioning," IEEE Trans. Signal Process., vol. 64, no. 15 , pp. 3895-3908, Aug. 2016.

[47] L. Huang, A. D. Joseph, B. Nelson, B. I. P. Rubinstein, and J. D. Tygar, "Adversarial machine learning," in Proc. 4th ACM Workshop Secur. Artif. Intell. (AISec). New York, NY, USA: ACM, 2011, pp. 43-58.

[48] L. Li, W. Chu, J. Langford, and X. Wang, "Unbiased offline evaluation of contextual-bandit-based news article recommendation algorithms," in Proc. WSDM. New York, NY, USA: ACM, 2011, pp. 297-306.

[49] I. Jolliffe, Principal Component Analysis. Hoboken, NJ, USA: Wiley, 2002.

[50] D. Michie, D. J. Spiegelhalter, C. C. Taylor, and J. Campbell, Eds., Machine Learning, Neural and Statistical Classification. Upper Saddle River, NJ, USA: Ellis Horwood, 1994.

[51] S. Escalera, O. Pujol, and P. Radeva, "Error-correcting ouput codes library," J. Mach. Learn. Res., vol. 11, pp. 661-664, Mar. 2010.

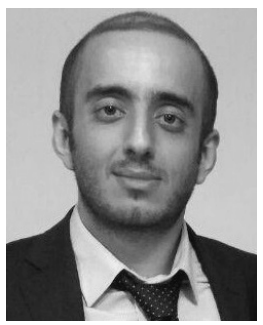

Mohammadreza Mohaghegh Neyshabouri received the B.S. degree in electrical engineering from the Sharif University of Technology, Tehran, Iran, in 2014. He is currently pursuing the M.S. degree in electrical and electronics engineering with Bilkent University, Ankara, Turkey.

His current research interests include statistical signal processing, online learning, and optimization. 


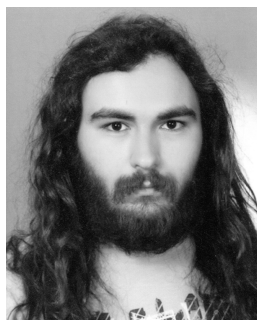

Kaan Gokcesu received the B.S. and M.S. degrees in electrical and electronics engineering from Bilkent University, Ankara, Turkey, in 2015 and 2017 , respectively. He is currently pursuing the $\mathrm{Ph} . \mathrm{D}$. degree in electrical engineering with the Massachusetts Institute of Technology, Cambridge, MA, USA.

His current research interests include online learning, adaptive filtering, big data, anomaly detection, and optimization.

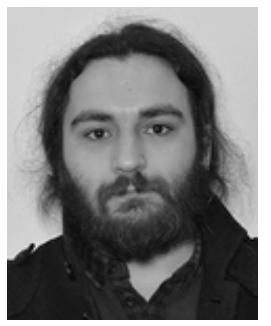

Hakan Gokcesu received the B.S. degree in electrical and electronics engineering from Bilkent University, Ankara, Turkey, in 2015. He is currently pursuing the M.S. degree in communication systems with data analytics specialization with the École Polytechnique Fédérale de Lausanne, Lausanne, Switzerland.

His current research interests include the electrical engineering/computer science and applied mathematics, including machine learning, optimization, big data, online learning, expert selection, and bandit problems.

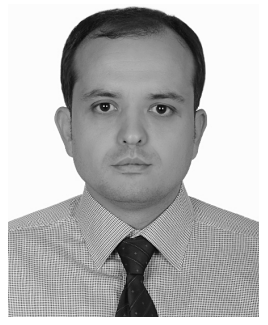

Huseyin Ozkan received the B.Sc. degree in electrical engineering and mathematics from Boğaziçi University, Istanbul, Turkey, the M.Sc. degree in electrical engineering from Boston University, Boston, MA, USA, and the Ph.D. degree in electrical engineering from Bilkent University, Ankara, Turkey.

He was a Post-Doctoral Research Associate of vision and computational neuroscience with the Massachusetts Institute of Technology, Cambridge, MA, USA. He is currently a full-time Faculty Member of electronics engineering with Sabancı University, Istanbul, Turkey, where he is also with the Faculty of Engineering and Natural Sciences. His current research interests include machine learning, signal processing, computer vision, and computational neuroscience.

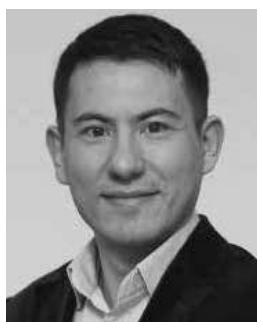

Suleyman Serdar Kozat (A'10-M'11-SM'11) received the B.S. degree (Hons.) from Bilkent University, Ankara, Turkey, and the M.S. and Ph.D. degrees in electrical and computer engineering from the University of Illinois at Urbana-Champaign, Urbana, IL, USA.

$\mathrm{He}$ was a Research Associate with the Cryptography and Anti-Piracy Group, Microsoft Research, Redmond, WA, USA. He joined the IBM Thomas J. Watson Research Center, Yorktown Heights, NY, USA, as a Research Staff Member, and later, he became a Project Leader of the Pervasive Speech Technologies Group, where he focused on problems related to statistical signal processing and machine learning. $\mathrm{He}$ is currently an Associate Professor with the Electrical and Electronics Engineering Department, Bilkent University. He has co-authored over 120 papers in refereed high-impact journals and conference proceedings. He holds several patent inventions (currently used in several different Microsoft and IBM products, such as MSN and ViaVoice). He also holds several patent inventions due to his research accomplishments with the IBM Thomas J. Watson Research Center and Microsoft Research. His current research interests include cyber security, anomaly detection, big data, data intelligence, adaptive filtering, and machine learning algorithms for signal processing. 